\title{
Zooming in on eV-MeV scale sterile neutrinos in light of neutrinoless double beta decay
}

\author{
Tapoja Jha, ${ }^{1,2,3,{ }^{*}}$ Sarif Khan $\odot,{ }^{4, \dagger}$ Manimala Mitra, ${ }^{1,2, *}$ and Ayon Patra $\odot^{5, \S}$ \\ ${ }^{1}$ Institute of Physics, Sachivalaya Marg, Bhubaneswar, Odisha 751005, India \\ ${ }^{2}$ Homi Bhabha National Institute, Training School Complex, Anushakti Nagar, Mumbai 400085, India \\ ${ }^{3}$ School of Physical Sciences, Indian Association for the Cultivation of Science, \\ $2 A$ and 2B Raja S.C. Mullick Road, Kolkata 700 032, India \\ ${ }^{4}$ Institüt für Theoretische Physik, Georg-August-Universität Göttingen, \\ Friedrich-Hund-Platz 1, 37077 Göttingen, Germany \\ ${ }^{5}$ Division of Physics, School of Advanced Sciences, VIT University, Chennai Campus, \\ Chennai 600127, India
}

(Received 19 July 2021; accepted 15 January 2022; published 1 February 2022)

\begin{abstract}
The existence of light sterile neutrinos, as predicted in several models, can help to explain a number of observations starting from dark mater to recent anomalies in short baseline experiments. In this paper, we consider two models-left-right symmetric Zee model and extended seesaw model - that can naturally accommodate the presence of light sterile neutrinos in the $\mathrm{eV}$ to $\mathrm{MeV}$ mass scale. We perform a detailed study on the neutrinoless double beta decay process which receives major contributions from diagrams involving these light sterile neutrinos. Considering a number of theoretical and experimental constraints, including light neutrino masses and mixings, unitarity of the mixing matrix, etc., we compare our predicted values of the half-life of neutrinoless double beta decay with the experimental limits. This can put significant constraints on the neutrino mass, active-sterile neutrino mixing, and several other important parameters in these models.
\end{abstract}

DOI: 10.1103/PhysRevD.105.035001

\section{INTRODUCTION}

The Standard Model (SM) of particle physics, despite its major successes, is unable to explain the observed light neutrino mass splittings and their mixings, which provides a strong motivation to invoke beyond the Standard Model (BSM) physics. The two observed neutrino mass splittings are $\Delta m_{12}^{2} \sim 10^{-5} \mathrm{eV}^{2},\left|\Delta m_{13}^{2}\right| \sim 10^{-3} \mathrm{eV}^{2}$ while the best-fit values of the neutrino mixing angles are $\theta_{12} \sim 34^{\circ}$, $\theta_{23} \sim 48^{\circ}$, and $\theta_{13} \sim 8^{\circ}$ [1]. Although neutrinos are massless in SM, a number of BSM theories have been proposed that successfully explain neutrino masses and mixings. One of the most appealing frameworks to generate Majorana masses of light neutrinos is via seesaw, where the dimension-5 lepton number violating operator generates the mass term after electroweak symmetry breaking [2-8].

\footnotetext{
*tapoja.phy@gmail.com

sarif.khan@uni-goettingen.de

*manimala@iopb.res.in

§ayon@okstate.edu
}

Published by the American Physical Society under the terms of the Creative Commons Attribution 4.0 International license. Further distribution of this work must maintain attribution to the author(s) and the published article's title, journal citation, and DOI. Funded by SCOAP.
The type-I seesaw serves as the most economical framework, as the model in addition to the SM particles are minimally extended by gauge singlet right-handed neutrinos. Another popular class of mechanism is the radiative mass generation [9-13], where neutrino mass is generated via a loop effect. In this work, we have considered a variation of the type-I seesaw model referred as the extended seesaw model $[14,15]$ and a left-right symmetric extension of radiative neutrino mass model [16-19].

The type-I seesaw model is the most economical, as the SM particle content is expanded with at least two heavy gauge singlet right-handed neutrinos which participate in light neutrino mass generation via the seesaw mechanism. However, the drawback of this simplest model is that the mixing of these right-handed neutrinos with SM neutrinos is tightly constrained by an $\mathrm{eV}$ light neutrino mass constraint, making the detection prospect of these righthanded neutrinos at experiments challenging. In extended seesaw, as the name suggests, more singlet neutrinos with large mixings are introduced with the possibility that some of them remain light and can be detected in experiments. The other popular mechanism for neutrino mass generation is through loop-induced processes. One of the simplest examples of this process is realized in the Zee model where the introduction of a doublet scalar and a charged singlet scalar can generate neutrino masses at the one-loop level. 
Although the simplest form of the Zee model [20] cannot satisfy neutrino oscillation data [21-23], its left-right symmetric extension however is consistent with experimental observations $[18,24]$. Here, the Majorana masses of the left-handed and right-handed neutrinos are generated at the one-loop level, while the Dirac mass term arises at the tree level from the Yukawa interactions. Finally, the light neutrino masses are obtained by a type- 1 seesaw mechanism but with the exception that the right-handed neutrinos can be light and hence offer better detection prospects.

Both of the above mentioned models can accommodate light right-handed neutrinos with masses ranging in the $\mathrm{eV}$ to $\mathrm{MeV}$ scale. An eV scale sterile neutrino is well motivated, as this can explain the Liquid Scintillator Neutrino Detector (LSND) anomaly [25-28]. Recently, this anomaly has also been favored by the MiniBooNE Collaboration [29], but at the same time the data have been disfavored by the KARMEN [30] and MINOS [31] observations. These issues may be finally tackled by the upcoming DUNE experiment $[32,33]$. Further hints regarding the presence of an $\mathrm{eV}$ scale sterile neutrino comes from the reactor antineutrino anomaly [34,35] and the Gallium anomaly [36,37]. A keV scale sterile neutrino can be an excellent candidate for warm dark matter. Several disagreements between the cosmological observations and the $N$ body simulations of structure formations can be solved by introducing a keV scale warm dark matter candidate [38]. The presence of an $\mathrm{MeV}$ sterile neutrino, on the other hand, can produce several observable astrophysical signals, such as its effect on the cosmic microwave background spectrum [39] and by producing X-ray photons which may be observable in satellite based X-ray experiments.

If the right-handed neutrinos are Majorana particles, they can give rise to additional contributions to the neutrinoless double beta decay $(0 \nu \beta \beta)$ process. The $0 \nu \beta \beta$ process is the transition $(A, Z) \rightarrow(A, Z+2)+2 e^{-}$with no neutrino being emitted [40-42]. The process is lepton number violating $[43,44]$. Depending on the mixing of the righthanded neutrinos with active neutrinos in type-I/extended seesaw, or the interaction of these right-handed neutrinos with the right-handed gauge boson in the left-right symmetric extension, these right-handed neutrino states may give significant contributions in the $0 \nu \beta \beta$ process compared to the three SM neutrino contributions and thus opening up the scope of detection of these Majorana neutrinos via a $0 \nu \beta \beta$ process. A number of experiments have searched for this process, and the nonobservation of the signal has given bounds on the half-life $T_{1 / 2}^{0 \nu}$ of $0 \nu \beta \beta$ [45-48]. The limit obtained on $T_{1 / 2}^{0 \nu}$ for ${ }^{76} \mathrm{Ge}$ is $T_{1 / 2}^{0 \nu}>8.0 \times 10^{25}$ year from GERDA-II [45], whereas at 90\% C.L., the KamLAND-Zen experiment has set a more stringent lower limit on the halflife of the ${ }^{136} \mathrm{Xe}$ isotope as $T_{1 / 2}^{0 \nu}>1.07 \times 10^{26}$ year [46].

In the present work, we consider the left-right symmetric Zee model (LRS Zee) and extended seesaw model that naturally accommodate light scale sterile neutrinos with masses $\sim \mathrm{eV}$ to $\mathrm{MeV}$. Our main goal is to study the $0 \nu \beta \beta$ phenomenology for these two models. The left-right symmetric extension of the Zee model $[18,24]$ presents a unique scenario where the model can be tested at the collider experiments as well as the neutrino experiments. It may have observable signals at the hadron $[18,24]$ and lepton colliders [19] and most notably can be accessed at the very early stage run of the upcoming $e^{+} e^{-}$colliders. In addition to satisfying all the neutrino mass and mixing constraints, this model can also give rise to several new $0 \nu \beta \beta$ processes which can significantly enhance the decay rate. This results in a marked decrease in the half-life of the $0 \nu \beta \beta$ decay process in this model. We study the variation of $T_{1 / 2}^{0 \nu}$ with respect to different model parameters and identify three of them which are most significant. These three parameters are the lightest neutrino mass, the Dirac $C P$ phase, and the mixing angle between the left and right gauge bosons. By varying these parameters, we identify the regions which can be ruled out from the experimental limits on the half-life of the $0 \nu \beta \beta$ process. In the case of extended seesaw mechanism, we first give an approximate analysis considering only a one-generation right-handed neutrino and one-generation active neutrino. Subsequently, we present a realistic analysis of the half-life with threegeneration active neutrinos and six-generation right-handed neutrinos. We have considered all the constraints arising both from theory and experiments. For the active neutrinos, we have considered bounds on the mass-square differences, three mixing angles in agreement with neutrino oscillation data [1], and the limit on the sum of the masses of active neutrinos which comes from the Planck satellite experiment [49]. We have ensured a mass hierarchy among these active and right-handed neutrinos to validate the seesaw approximation for this model, as well as have considered constraints from nonunitarity [50,51]. We have calculated the $0 \nu \beta \beta$ decay contribution considering all the required model parameters which pass all the aforementioned constraints and have checked if the predicted contribution satisfies the corresponding experimental limits [46,52].

The paper is organized in the following way. In Sec. II, we present a detailed study of the $0 \nu \beta \beta$ process in the LRS Zee model. This is followed by Sec. III, where we give a detailed description of the extended seesaw scenario and the analysis of the model with respect to many theoretical and experimental aspects, e.g., neutrinuo oscillation data, $0 \nu \beta \beta$ decay, unitarity, and others. Finally, we present our conclusions in Sec. IV.

\section{LEFT-RIGHT SYMMETRIC ZEE MODEL AND ANALYSIS}

The Zee model [20] is one of the simplest extensions of the Standard Model (SM) which can explain the origin of neutrino mass. By extending the SM framework with an extra doublet and a charged singlet scalar, neutrino masses can be successfully generated at the one-loop level. 
This simplest form of the Zee model, though, is found to be incompatible with the neutrino experimental data [21-23,53], and one needs to extend it further in order to get a viable scenario to explain all the neutrino oscillation constraints. The left-right symmetric extension of the Zee model $[18,24]$ provides an alternate model framework which can easily explain the neutrino oscillation data as well as provide interesting flavor violating signals and unique collider signatures.

The pair production and decay of the singly charged Higgs boson can produce final states with two charged leptons (with either same or different flavors) and missing transverse energy. This process has been studied in the context of the Large Hadron Collider (LHC) in Ref. [18,24] and for the International Linear Collider (ILC) and the Compact Linear Collider (CLIC) in Ref. [19]. The charged singlet scalar pair-production cross section at hadron colliders is quite small and is dominated by the photon mediated process, while it can become significantly larger in the electron-positron collider due to the right-handed neutrino mediated $t$ channel diagram. Thus, the ILC and CLIC experiments may be able to observe such a particle with very low integrated luminosity of only $1-3 \mathrm{fb}^{-1}$, see [19] for details. Below, we present a brief discussion on the model.

\section{A. Model}

We consider an extended Zee Model with the gauge group $S U(3)_{C} \times S U(2)_{L} \times S U(2)_{R} \times U(1)_{B-L}$. The fermion sector in this model contains both left-handed and right-handed fermion doublets. This naturally includes the presence of three right-handed neutrinos in addition to the SM fermions. The Yukawa Lagrangian in this model is given as $[18,19,24]$

$$
\begin{aligned}
\mathcal{L}_{Y}= & Y_{i j}^{q 1} \bar{Q}_{L i} \Phi Q_{R j}+Y_{i j}^{q 2} \bar{Q}_{L i} \tilde{\Phi} Q_{R j}+Y_{i j}^{l 1} \bar{l}_{L i} \Phi l_{R j} \\
& +Y_{i j}^{l 2} \bar{l}_{L i} \tilde{\Phi} l_{R j}+\lambda_{L_{i j}} l_{L i}^{T} i \tau_{2} l_{L j} \delta^{+}+\lambda_{R_{i j}} l_{R i}^{T} i \tau_{2} l_{R j} \delta^{+} \\
& + \text {H.c. }
\end{aligned}
$$

where $\Phi$ and $\delta$ are the bidoublet and charged singlet scalars with $Y$ and $\lambda$ being their respective Yukawa coupling matrices. The Majorana masses of all the neutrinos are generated at the one-loop level, and as a result, they remain quite light. In order to understand the neutrino sector, we also need to understand the scalar sector of the model.

\section{Charged scalar spectrum}

The minimal Higgs sector in this model consists of a bidoublet, two doublets, and a charged singlet field given as

$$
\begin{gathered}
H_{R}(1,1,2,1)=\left(\begin{array}{c}
H_{R}^{+} \\
H_{R}^{0}
\end{array}\right), \quad H_{L}(1,2,1,1)=\left(\begin{array}{c}
H_{L}^{+} \\
H_{L}^{0}
\end{array}\right), \\
\Phi(1,2,2,0)=\left(\begin{array}{ll}
\phi_{1}^{0} & \phi_{2}^{+} \\
\phi_{1}^{-} & \phi_{2}^{0}
\end{array}\right), \quad \delta(1,1,1,2)=\delta^{+} .
\end{gathered}
$$

The doublet $H_{R}$ is responsible for the right-handed symmetry breaking, while $H_{L}$ is required for parity conservation. The bidoublet $\Phi$ helps break the electroweak symmetry and generates the SM particle masses. The charged scalar field $\delta$, similar to the Zee model, helps generate the neutrino Majorana masses at the one-loop level. The nonzero vacuum expectation values (VEVs) of the neutral scalar fields are given as

$\left\langle H_{R}^{0}\right\rangle=v_{R}, \quad\left\langle H_{L}^{0}\right\rangle=v_{L}, \quad\left\langle\phi_{1}^{0}\right\rangle=v_{1}, \quad\left\langle\phi_{2}^{0}\right\rangle=v_{2}$,

with the effective electroweak (EW) VEV given as $v_{\mathrm{EW}}=$ $\sqrt{v_{1}^{2}+v_{2}^{2}+v_{L}^{2}}$. The $S U(2)_{R} \times U(1)_{B-L}$ symmetry is broken down to $U(1)_{Y}$ as the neutral component of the right-handed doublet $H_{R}^{0}$ acquires a VEV $v_{R}$. The bidoublet VEVs $v_{1}$ and $v_{2}$ are required to generate the quark and charged lepton masses and Cabibbo-Kobayashi-Maskawa (CKM) mixing angles. We can easily choose one of the bidoublet VEVs to be much smaller than the other without loss of generality. Also, since $v_{L}$ does not contribute to the fermion masses, we choose it to be small as well. Thus, the hierarchy of the VEVs can be given as

$$
v_{R} \gg v_{1}>v_{2}, v_{L} .
$$

Thus, $v_{1}$ gives the largest contribution to the EW VEV. The charged Higgs bosons play important roles in the generation of the one-loop neutrino masses as their masses and mixings become important parameters in the expression for the radiative neutrino Majorana masses. So, before we study the neutrino mass generation mechanism, it is important to define the mass basis for the charged Higgs bosons. There are in total five charged Higgs states which mix to give five mass eigenstates through the rotation

$$
\left(\begin{array}{c}
\phi_{1}^{-*} \\
\phi_{2}^{+} \\
H_{R}^{+} \\
H_{L}^{+} \\
\delta^{+}
\end{array}\right)=V\left(\begin{array}{c}
H_{1}^{+} \\
H_{2}^{+} \\
H_{3}^{+} \\
G_{1}^{+} \\
G_{2}^{+}
\end{array}\right)
$$

where $V$ is the $5 \times 5$ charged Higgs mixing matrix which can be written as 


$$
V=\left(\begin{array}{lllll}
V_{11} & V_{12} & V_{13} & V_{14} & V_{15} \\
V_{21} & V_{22} & V_{23} & V_{24} & V_{25} \\
V_{31} & V_{32} & V_{33} & V_{34} & V_{35} \\
V_{41} & V_{42} & V_{43} & V_{44} & V_{45} \\
V_{51} & V_{52} & V_{53} & V_{54} & V_{55}
\end{array}\right) .
$$

There are three physical charged scalars $H_{1}^{+}, H_{2}^{+}, H_{3}^{+}$ which will contribute to the neutrino masses and two Goldstone states $G_{1}^{+}, G_{2}^{+}$which are eaten up by the $W_{R}$ and $W$ bosons as their longitudinal degrees of freedom. In this model, largely the $H_{R}^{+}$and the $\phi_{1}^{-*}$ become the Goldstone bosons, while the other three charged states mix to form the physical Higgs bosons. This implies that in the first and third rows of the mixing matrix only the $V_{15}$ and $V_{34}$ elements are almost unity, while the others are small $\left(\lesssim 10^{-3}\right)$. We have considered a benchmark point where three charged Higgs have masses as $473 \mathrm{GeV}$, $1.84 \mathrm{TeV}$, and $15.2 \mathrm{TeV}$, respectively. Here, the two lighter states are composed of a mixture ${ }^{1}$ of the charged singlet $\left(\delta^{+}\right)$and left-handed charged Higgs $\left(H_{L}^{+}\right)$, while the heaviest state is coming primarily from the second bidoublet $\left(\phi_{2}^{+}\right)$. Constraints from the flavor violating process require the mass of the second bidoublet scalar $\phi_{2}^{+}$to be heavier than $15 \mathrm{TeV}$ [54-59]. This further implies that the $V_{23}$ element in the second row of the $V$ matrix is close to one, while others are quite small. Finally, since the charged singlet $\delta^{+}$can have considerable mixing with the lefthanded charged Higgs boson $H_{L}^{+}$, the $V_{41}, V_{42}, V_{51}$, and $V_{52}$ elements can become significant. This gives us a good idea of which elements in matrix $V$ can play important roles in the neutrino mass generation.

\section{Fermion mass and mixings}

Applying a simple rotation of the bidoublet scalar fields to

$$
h_{1}^{0}=\frac{v_{1} \phi_{1}^{0}+v_{2} \phi_{2}^{0}}{\sqrt{v_{1}^{2}+v_{2}^{2}}}, \quad h_{2}^{0}=\frac{v_{2} \phi_{1}^{0}-v_{1} \phi_{2}^{0}}{\sqrt{v_{1}^{2}+v_{2}^{2}}}
$$

along with a redefinition of the Yukawa couplings as

$$
\begin{aligned}
Y^{q}=\frac{1}{v_{1}^{\prime}}\left(Y^{q 1} v_{1}+Y^{q 2} v_{2}\right), & \tilde{Y}^{q}=\frac{1}{v_{1}^{\prime}}\left(Y^{q 1} v_{2}+Y^{q 2} v_{1}\right), \\
Y^{l}=\frac{1}{v_{1}^{\prime}}\left(Y^{l 1} v_{1}+Y^{l 2} v_{2}\right), & \tilde{Y}^{l}=\frac{1}{v_{1}^{\prime}}\left(Y^{l 1} v_{2}+Y^{l 2} v_{1}\right),
\end{aligned}
$$

gives the fermion masses in this model to be

\footnotetext{
${ }^{1}$ We consider two extreme cases-maximal and minimal mixings.
}

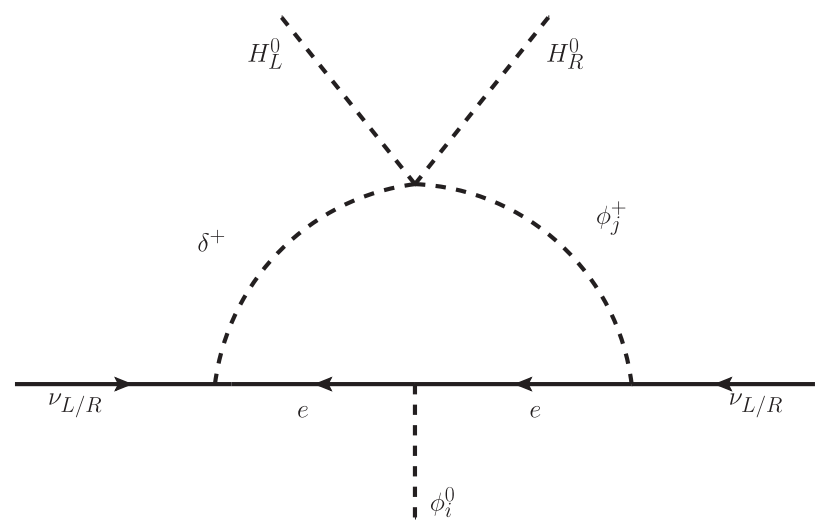

FIG. 1. Neutrino Majorana mass generation at one-loop in the LRS Zee model.

$$
\begin{aligned}
M_{u} & =Y^{q} v_{1}^{\prime}, & M_{d} & =\tilde{Y}^{q} v_{1}^{\prime}, \\
M_{l} & =\tilde{Y}^{l} v_{1}^{\prime}, & M_{\nu}^{D} & =Y^{l} v_{1}^{\prime} .
\end{aligned}
$$

Here, $M_{u}, M_{d}$, and $M_{l}$ are the up-type quark, down-type quark, and charged lepton mass matrices, while $M_{\nu}^{D}$ is the neutrino Dirac mass matrix.

The neutrino sector consists of three left-handed and three right-handed neutrinos. The absence of triplet scalars in the model prevents us from writing a Majorana mass term for the neutrinos. All the neutrino Majorana masses here are generated at the one-loop level and hence are quite small. The lightest right-handed neutrino mass ranges from a few $\mathrm{eV}$ to a few hundred $\mathrm{eV}$, and the other right-handed neutrinos also remain lighter than an MeV. The Dirac masses are thus required to be quite small as well, so as to satisfy the experimentally observed neutrino masses and mixings. ${ }^{2}$ The one-loop Feynman diagram for the generation of neutrino Majorana masses is given in Fig. 1. The corresponding expressions for the neutrino Majorana masses in this case are given as [24]

$$
\begin{aligned}
\left(M_{\nu}^{L}\right)^{\alpha \gamma}= & \frac{1}{4 \pi^{2}} \lambda_{L}^{\prime \alpha \beta} m_{e_{\beta}} \sum_{i=1}^{3} \log \left(\frac{M_{h_{i}}^{2}}{m_{e_{\beta}}^{2}}\right) \\
& \times V_{5 i}\left[\left(Y_{l}^{\dagger}\right)^{\beta \gamma} V_{2 i}^{*}-\left(\tilde{Y}_{l}^{\dagger}\right)^{\beta \gamma} V_{1 i}^{*}\right]+\alpha \leftrightarrow \gamma, \\
\left(M_{\nu}^{R}\right)^{\alpha \gamma}= & \frac{1}{4 \pi^{2}} \lambda_{R}^{\alpha \beta} m_{e_{\beta}} \sum_{i=1}^{3} \log \left(\frac{M_{h_{i}}^{2}}{m_{e_{\beta}}^{2}}\right) \\
& \times V_{5 i}\left[\left(Y_{l}\right)^{\beta \gamma} V_{1 i}^{*}-\left(\tilde{Y}_{l}\right)^{\beta \gamma} V_{2 i}^{*}\right]+\alpha \leftrightarrow \gamma .
\end{aligned}
$$

In the above, $\lambda_{L / R}^{\prime \alpha \beta}=\lambda_{L / R}^{\alpha \beta}-\lambda_{L / R}^{\beta \alpha}, m_{e_{\beta}}$ and $M_{h_{i}}$ are the charged lepton and Higgs boson masses, respectively, and $V_{i j}$ are the charged Higgs boson mixings given in Eq. (5). For our calculations, we consider the case with $\lambda_{L}^{\prime}=0$ as

\footnotetext{
${ }^{2}$ Detailed analysis of the neutrino sector of the LRS Zee model is presented in Ref. [19].
} 


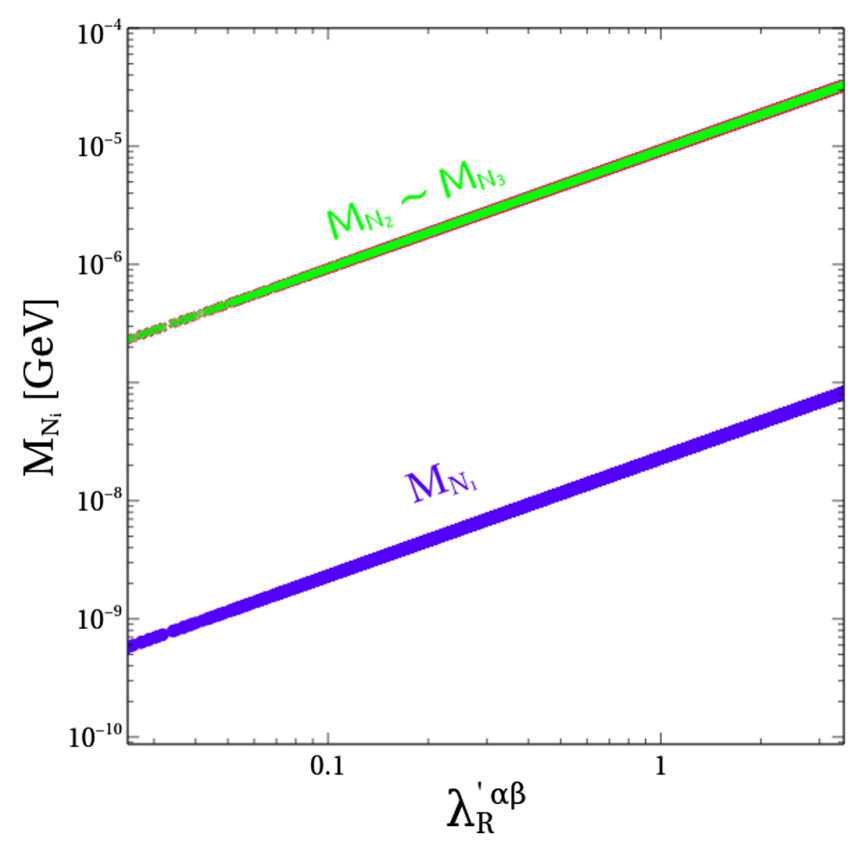

FIG. 2. Right-handed neutrino Majorana masses in the LRS Zee model [19].

was discussed in [19]. This thus gives us a $6 \times 6$ neutrino mass matrix given as

$$
M_{\nu}=\left(\begin{array}{cc}
0 & M_{\nu}^{D} \\
M_{\nu}^{D^{T}} & M_{\nu}^{R}
\end{array}\right) .
$$

We thus have a scenario that is very similar to the type-I seesaw mechanism, i.e., the light and heavy neutrino mass matrix after pursuing a block-diagonalization becomes

$$
\mathcal{M}_{\nu}=-M_{\nu}^{D} M_{\nu}^{R-1} M_{\nu}^{D T}, \quad \mathcal{M}_{n}=M_{\nu}^{R} .
$$

The neutrino rotation matrix, taking it from flavor to mass eigenstates, will be a $6 \times 6$ matrix which we can write as

$$
\mathcal{V}=\left(\begin{array}{ll}
\mathbb{U} & \mathbb{S} \\
\mathbb{T} & \mathbb{V}
\end{array}\right)
$$

such that

$$
\mathcal{V}^{T} M_{\nu} \mathcal{V}=\left(\begin{array}{cc}
\hat{M}_{\nu} & 0 \\
0 & \hat{M}_{N}
\end{array}\right) .
$$

Here, $\hat{M}_{\nu}=\operatorname{diag}\left(m_{1}, m_{2}, m_{3}\right)$ and $\hat{M}_{N}=\operatorname{diag}\left(M_{1}, M_{2}, M_{3}\right)$ are diagonal matrices consisting of the light and heavy neutrino masses, respectively.

Figure 2 shows a plot of the Majorana masses of the right-handed neutrinos as generated at the one-loop level in this model. The plot shows the variation of the eigenvalues of the right-handed neutrino Majorana mass matrix [elements of the matrix given in Eq. (10)] as a function of its coupling with the charged singlet scalar. Here, the lightest charged Higgs boson has a mass of $473 \mathrm{GeV}$ and primarily consists of charged singlet $\delta$. As $\lambda_{R}^{\prime}$ is varied from 0.1 to 3 , the lightest right-handed neutrino mass $M_{N_{1}}$ varies from 3 to $80 \mathrm{eV}$, while $M_{N_{2,3}}$ remain almost degenerate and in the sub-MeV scale.

In order to understand the values of the masses, we need to take a closer look at the one-loop Majorana mass expression given in Eq. (10). The expression shows that the Majorana masses are proportional to the charged lepton masses, the Yukawa couplings $Y_{l}$ and $\widetilde{Y}_{l}$, and the charged Higgs boson mixings $V_{5 i}, V_{2 i}, V_{1 i}$, and logarithmically dependent on the charged Higgs boson masses. Let us denote the charged lepton masses as $m_{e}, m_{\mu}$, and $m_{\tau}$ for electron, muon, and tau lepton, respectively. The elements of the Yukawa coupling matrix $Y_{l}$ generating the neutrino Dirac mass term are extremely small and can be safely neglected. The $\widetilde{Y}_{l}$ matrix gives rise to the charged lepton masses and is chosen to be diagonal with $\tilde{Y}_{l_{11}} \sim 10^{-6}$, $\tilde{Y}_{l_{22}} \sim 10^{-4}$, and $\tilde{Y}_{l_{33}} \sim 10^{-2}$. From our previous discussion, we know that only the elements $V_{51}, V_{52}, V_{15}$, and $V_{23}$ can be large $(\sim 1)$, while the other couplings are quite small $\left(\lesssim 10^{-3}\right)$. This shows that for any of the Majorana mass terms, only the product of a large coupling along with a small coupling will appear. This introduces a suppression of at least $10^{-3}$ into the mass terms. Let us now look at each of the Majorana mass terms $\left(M_{\nu}^{R}\right)^{\alpha \gamma}$. These terms are symmetric in $\alpha \gamma$, and hence, the diagonal terms $(\alpha=\gamma)$ vanish as $\lambda_{R}^{\prime}$ is an antisymmetric matrix. Considering the off-diagonal terms, for $\left(M_{\nu}^{R}\right)^{12}$, only the combinations $\tilde{Y}_{l_{11}} m_{e}$ and $\tilde{Y}_{l_{22}} m_{\mu}$ will contribute. This gives a value of $\left(M_{\nu}^{R}\right)^{12} \sim 10^{-8}$ for a heavy charged Higgs boson mass of $15 \mathrm{TeV}$ and $\lambda_{R}^{\prime} \sim 1$. For $\left(M_{\nu}^{R}\right)^{13}$, the contributions come from the combinations $\tilde{Y}_{l_{11}} m_{e}$ and $\tilde{Y}_{l_{33}} m_{\tau}$, while for $\left(M_{\nu}^{R}\right)^{23}$ the contributions come from the combinations $\tilde{Y}_{l_{22}} m_{\mu}$ and $\tilde{Y}_{l_{33}} m_{\tau}$. For both these terms, the leading contribution comes from the $\tau$ lepton contribution, and hence, they are almost degenerate with $\left(M_{\nu}^{R}\right)^{13} \approx\left(M_{\nu}^{R}\right)^{23} \sim 10^{-5}$ for a heavy charged Higgs boson mass of $15 \mathrm{TeV}$ and $\lambda_{R}^{\prime} \sim 1$.

Below, we discuss the contribution of the right-handed neutrinos in $0 \nu \beta \beta$ decay.

\section{B. Diagrams and amplitudes of $0 \nu \beta \beta$ transition}

Contrary to most seesaw models which contain the righthanded neutrinos of TeV scale mass, the LRS Zee model naturally accommodates $\mathrm{eV}-\mathrm{MeV}$ scale right-handed neutrinos, as has already been discussed in the previous section. Diagrams involving the right-handed neutrinos can thus significantly contribute to the $0 \nu \beta \beta$ processes, and this model gives us an excellent framework to study these effects.

The Feynman diagrams of all the possible contributions are presented in Fig. 3. For each diagram, we write its 


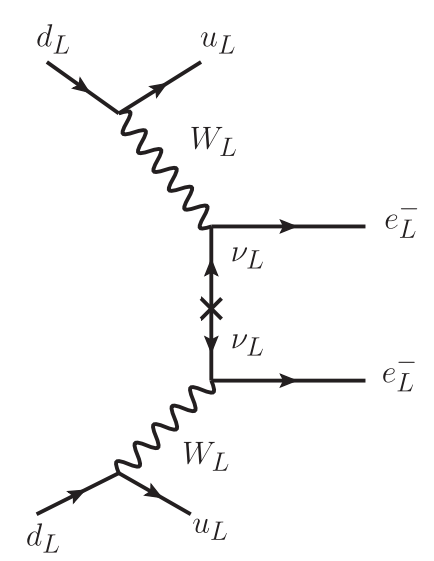

(a)

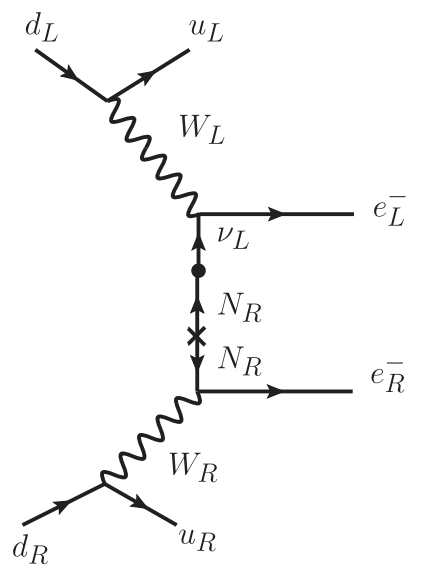

(e)

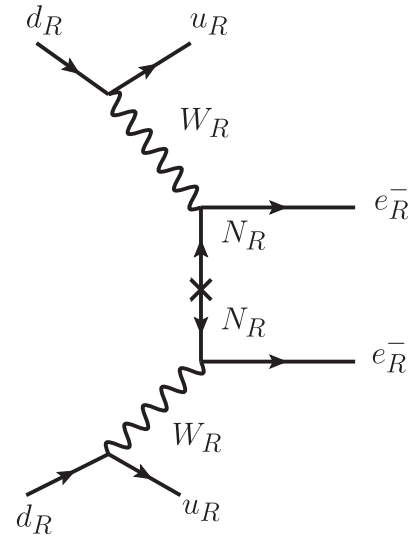

(b)

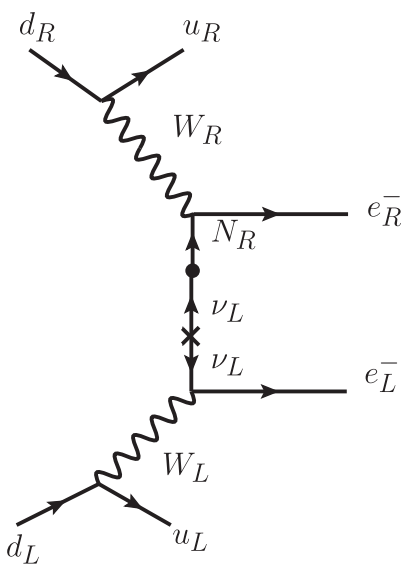

(f)

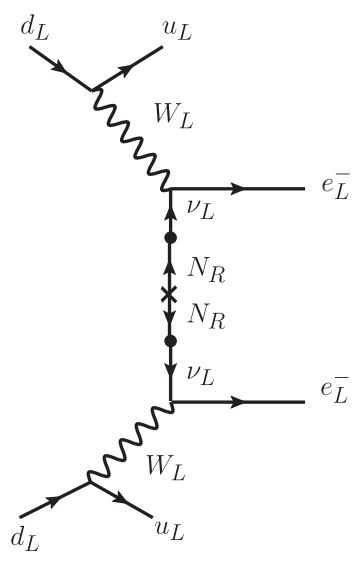

(c)

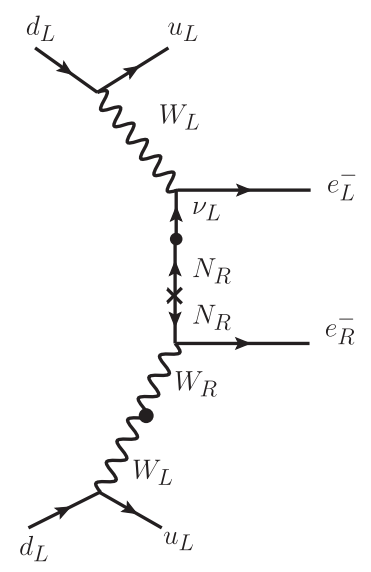

(g)

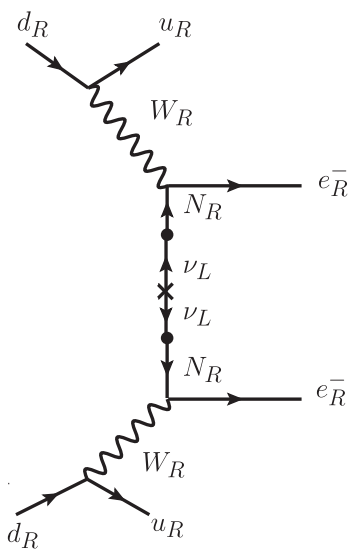

(d)

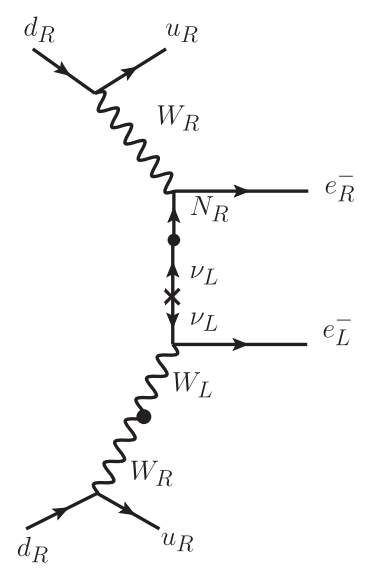

(h)

FIG. 3. Feynman diagrams of all possible $0 \nu \beta \beta$ processes in the LRS Zee model.

amplitude and identify the dimensionless parameter $\eta_{i}$ that will be used in the computation of the half life $\left(T_{1 / 2}^{0 \nu}\right)$ of the $0 \nu \beta \beta$ process. In the subsequent discussion, we refer to the mass eigenstates of SM neutrinos as "light" and the righthanded neutrinos as "heavy", as the right-handed neutrino states are heavier than the SM neutrinos.

(i) Light neutrino diagram: Fig. 3(a) corresponds to the light neutrino contribution. Its amplitude is given as

$$
A_{1} \simeq G_{F}^{2} \sum_{i} \mathbb{U}_{e i}^{2} \frac{m_{i}}{p^{2}}
$$

where $G_{F}$ is the Fermi constant, $p$ the momentum transfer at the leptonic vertex, and $i=1,2,3$ corresponds to the light neutrino mass eigenstates. The corresponding $\eta$ obtained in this case is given as

$$
\eta_{1}=\frac{1}{m_{e}} \sum_{i} \mathbb{U}_{e i}^{2} m_{i}
$$

(ii) Heavy neutrino diagrams: Fig. 3(b) corresponds to the heavy neutrino contribution. The heavy neutrinos in this model are composed of the right-handed neutrinos, but unlike other left-right models, they are quite light in this case with masses in the $\mathrm{eV}$ to $\mathrm{MeV}$ range. The Feynman amplitude and the corresponding $\eta$ from this diagram is given as

$$
\begin{aligned}
A_{2} & \simeq G_{F}^{2}\left(\frac{M_{W_{L}}}{M_{W_{R}}}\right)^{4} \sum_{i} \mathbb{V}_{e i}^{2} \frac{M_{i}}{p^{2}}, \\
\eta_{2} & =\frac{1}{m_{e}}\left(\frac{M_{W_{L}}}{M_{W_{R}}}\right)^{4} \sum_{i} \mathbb{V}_{e i}^{2} M_{i},
\end{aligned}
$$

where the summation is over the heavy neutrino eigenstates in this case.

(iii) Light-heavy neutrino mixing diagram: Figs. 3(c) and 3(d) correspond to the contributions due to the mixing between the light and heavy neutrinos. The Feynman amplitudes are given as 
$A_{3} \simeq G_{F}^{2} \sum_{i} \mathbb{S}_{e i}^{2} \frac{M_{i}}{p^{2}}, \quad A_{4} \simeq G_{F}^{2}\left(\frac{M_{W_{L}}}{M_{W_{R}}}\right)^{4} \sum_{i} \mathbb{T}_{e i}^{2} \frac{m_{i}}{p^{2}}$,

while the $\eta$ factors are

$$
\eta_{3}=\frac{1}{m_{e}} \sum_{i} \mathbb{S}_{e i}^{2} M_{i}, \quad \eta_{4}=\frac{1}{m_{e}}\left(\frac{M_{W_{L}}}{M_{W_{R}}}\right)^{4} \sum_{i} \mathbb{T}_{e i}^{2} m_{i} .
$$

(iv) $\lambda$ diagrams: Figs. 3(e) and 3(f) represent the processes mediated by the $W_{L}-W_{R}$ exchange. The Feynman amplitudes from each diagram can be easily combined to give us a final expression which is

$$
A_{\lambda} \simeq G_{F}^{2}\left(\frac{M_{W_{L}}}{M_{W_{R}}}\right)^{2} \sum_{i} \frac{\mathbb{U}_{e i} \mathbb{T}_{e i}^{*}+\mathbb{V}_{e i} \mathbb{S}_{e i}^{*}}{p}
$$

and the expression for the $\eta$ parameter is

$$
\eta_{\lambda}=\left(\frac{M_{W_{L}}}{M_{W_{R}}}\right)^{2} \sum_{i} \mathbb{U}_{e i} \mathbb{T}_{e i}^{*}+\mathbb{V}_{e i} \mathbb{S}_{e i}^{*}
$$

(v) $\eta$ diagrams: Figs. $3(\mathrm{~g})$ and $3(\mathrm{~h})$ are due to the $W_{L}-W_{R}$ mixing in this model and depend on the $W_{L}-W_{R}$ mixing angle $\theta_{L R}$. The Feynman amplitude combining the two diagrams can be written as

$$
A_{\eta}=G_{F}^{2} \tan \theta_{L R} \sum_{i} \frac{\mathbb{U}_{e i} \mathbb{\mathbb { T }}_{e i}^{*}+\mathbb{V}_{e i} \mathbb{S}_{e i}^{*}}{p}
$$

and the corresponding $\eta$ parameter is

$$
\eta_{\eta}=\tan \theta_{L R} \sum_{i} \mathbb{U}_{e i} \mathbb{T}_{e i}^{*}+\mathbb{V}_{e i} \mathbb{S}_{e i}^{*}
$$

The half-life for the $0 \nu \beta \beta$ process after combining the contributions from all these diagrams is then given as [60-63]

$$
\begin{aligned}
T_{1 / 2}^{0 \nu}= & {\left[G _ { 0 \nu } \left(\left|M_{\nu}^{0 \nu} \eta_{1}+M_{\nu}^{0 \nu} \eta_{3}\right|^{2}+\left|M_{\nu}^{0 \nu} \eta_{2}+M_{\nu}^{0 \nu} \eta_{4}\right|^{2}\right.\right.} \\
& \left.\left.+\left|M_{\lambda}^{0 \nu} \eta_{\lambda}+M_{\eta}^{0 \nu} \eta_{\eta}\right|^{2}\right)\right]^{-1},
\end{aligned}
$$

where $G_{0 \nu}$ is the phase space factor; $M_{\nu}^{0 \nu}, M_{\lambda}^{0 \nu}$, and $M_{\eta}^{0 \nu}$ are the nuclear matrix elements.

Now that we have the expression for the half-life for the $0 \nu \beta \beta$ processes, let us discuss some of the features of this framework which will help us understand the relative contribution arising from each of these diagrams. The right-handed neutrino masses being at the $\mathrm{eV}$ to $\mathrm{MeV}$ scale contribute significantly to these processes here, and hence, the diagrams involving $N_{R}$ become quite important.
The relative contributions from the diagrams are also highly dependent on the light-heavy neutrino mixings $(\mathbb{S}, \mathbb{T}$ ) with the $\lambda$ and $\eta$ diagrams becoming significant as this mixing increases. The gauge boson $\left(W_{L}-W_{R}\right)$ mixing is another important factor in these diagrams, and its value can determine which diagram gives a significant contribution to the $0 \nu \beta \beta$ decay process. Finally, since the $W_{R}$ boson mass is required to be quite large from experimental constraints [55-58,64-72], we have chosen it to be $5.5 \mathrm{TeV}$ here, and this results in a large suppression for all the diagrams with amplitudes involving the $\left(M_{W_{L}} / M_{W_{R}}\right)^{4}$ term.

The neutrino parameters in this model depend significantly on the masses and mixings of the charged scalars as can be seen quite clearly from Eq. (10). A close inspection of this equation also shows that the factor $V_{5 i}$ which is the mixing between the charged singlet and other charged Higgs states is quite important for the neutrino masses. As discussed earlier, the charged singlet Higgs $\delta^{+}$can only have significant mixing with the left-handed charged scalar $H_{L}^{+}$. We can thus approximately write

$$
\begin{aligned}
& H_{1}^{+}=\delta^{+} \cos \theta+H_{L}^{+} \sin \theta, \\
& H_{2}^{+}=-\delta^{+} \sin \theta+H_{L}^{+} \cos \theta,
\end{aligned}
$$

where $\mathrm{H}_{1}^{+}$and $\mathrm{H}_{2}^{+}$are the lightest and next-to-lightest charged Higgs bosons, respectively, with $\theta$ being the mixing angle. Clearly two extreme cases appear here

(i) maximal mixing with $\theta=\pi / 4$, denoted as $\mathrm{H}_{\max }$,

(ii) minimal mixing with $\theta=0$, denoted as $\mathrm{H}_{\min }$.

\section{Results}

To analyse the half-life of the $0 \nu \beta \beta$ process for germanium $\left({ }^{76} \mathrm{Ge}\right)$ and xenon $\left({ }^{136} \mathrm{Xe}\right)$ nuclei, we consider two cases of maximal and minimal mixing as described in the previous section. We consider a normal mass ordering among the SM neutrinos and use the Casas-Ibarra [73] parametrization to fit the latest neutrino oscillation data [1]. As the right-handed neutrino masses are quite small here, the mixing between them and the left-handed neutrinos, represented by the $\mathbb{S}$ and $\mathbb{T}$ matrices in Eq. (13), can become quite significant. ${ }^{3}$ For a fixed choice of the righthanded neutrino masses, the light-heavy neutrino $\left(\nu_{L}-N_{R}\right)$ mixing depends largely on the light neutrino masses. As the SM neutrino masses increase, the mass difference between the light and heavy neutrino states become smaller resulting in a larger mixing angle. Thus, the lightest neutrino mass $m_{\nu_{1}}$ is an important parameter for our analysis.

The other parameters which play significant roles in determining the value of $T_{1 / 2}^{0 \nu}$ are the $W_{L}-W_{R}$ mixing

\footnotetext{
${ }^{3}$ These terms still remain orders of magnitude smaller than the $\mathbb{U}$ and $\mathbb{V}$ matrices.
} 

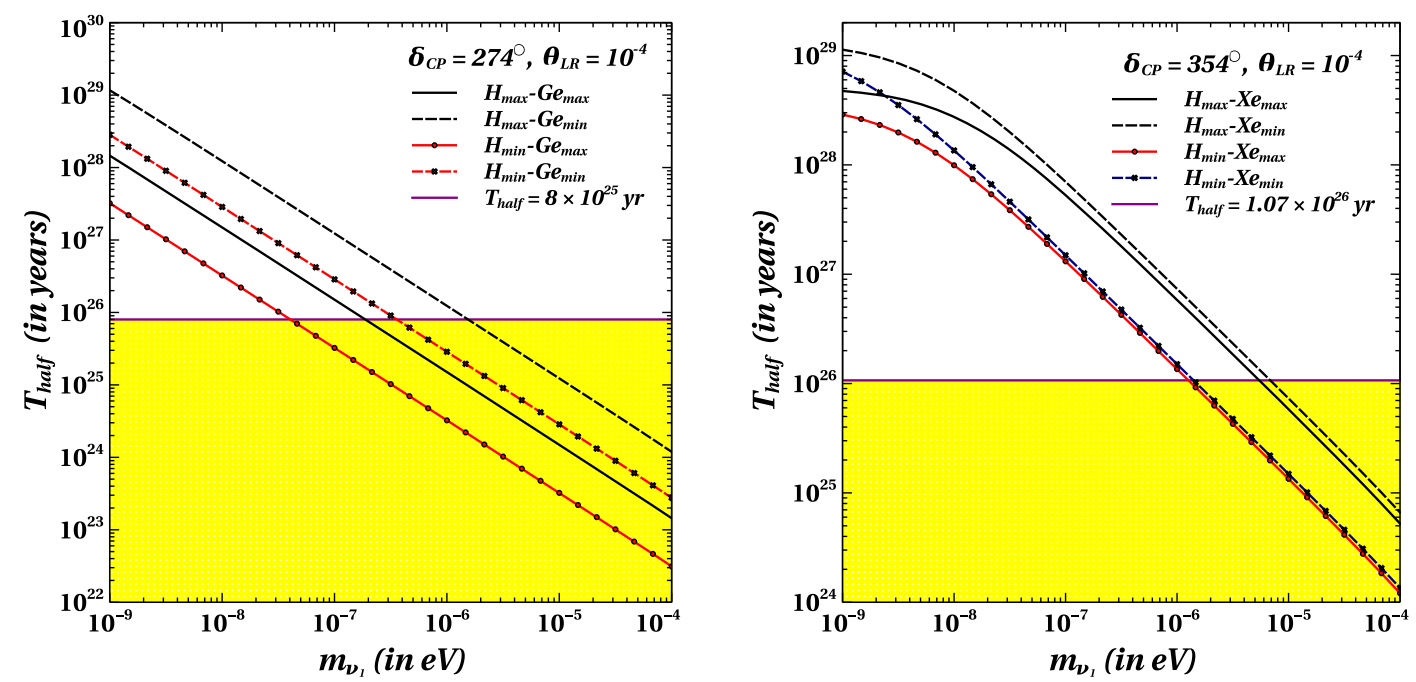

FIG. 4. Half-life of the $0 \nu \beta \beta$ process for ${ }^{76} \mathrm{Ge}$ and ${ }^{136} \mathrm{Xe}$ nuclei as a function of the lightest neutrino mass. The shaded region corresponds to $T_{1 / 2}^{0 \nu}<8.0 \times 10^{25}$ years for the left panel and $1.07 \times 10^{26}$ years for the right panel and disallowed by GERDA [45] and KamLAND-Zen [46], respectively.

angle $\left(\theta_{L R}\right)$ and the Dirac $C P$ phase $\left(\delta_{C P}\right)$ of the neutrino Pontecorvo-Maki-Nakagawa-Sakata (PMNS) matrix. The contribution from the $\eta$ diagram, directly proportional to $\tan \theta_{L R}$, can become substantial depending on this mixing angle. The value of $\delta_{C P}$, although directly not appear in any of the expressions, determines the neutrino parameters obtained from the Cassas-Ibarra parametrization. This has a significant consequence on the calculated value of $T_{1 / 2}^{0 \nu}$. The nuclear matrix elements (NMEs) for ${ }^{76} \mathrm{Ge}$ and ${ }^{136} \mathrm{Xe}$, which we adopt from [45,74-82], are equally important for the evaluation of $T_{1 / 2}^{0 \nu}$. We consider two cases, one with the maximum and another with the minimum values of the NMEs, and evaluate the half-life. For each nucleus $\left({ }^{76} \mathrm{Ge}\right.$ or $\left.{ }^{136} \mathrm{Xe}\right)$, we thus get four separate cases which are as follows:

(a) $\mathrm{H}_{\min }-\left({ }^{76} \mathrm{Ge} /{ }^{136} \mathrm{Xe}\right)_{\min }$ : Corresponds to the case where the Higgs boson mixing is minimum, and the minimum value for the ${ }^{76} \mathrm{Ge} /{ }^{136} \mathrm{Xe}$ NME has been used.

(b) $\mathrm{H}_{\max }-\left({ }^{76} \mathrm{Ge} /{ }^{136} \mathrm{Xe}\right)_{\min }$ : Corresponds to the case where the Higgs boson mixing is maximum, and the minimum value for the ${ }^{76} \mathrm{Ge} /{ }^{136} \mathrm{Xe} \mathrm{NME}$ has been used.

(iii) $\mathrm{H}_{\min }-\left({ }^{76} \mathrm{Ge} /{ }^{136} \mathrm{Xe}\right)_{\max }$ : Corresponds to the case where the Higgs boson mixing is minimum, and the maximum value for the ${ }^{76} \mathrm{Ge} /{ }^{136} \mathrm{Xe}$ NME has been used.

(iv) $\mathrm{H}_{\max }-\left({ }^{76} \mathrm{Ge} /{ }^{136} \mathrm{Xe}\right)_{\max }$ : Corresponds to the case where the Higgs boson mixing is maximum, and the maximum value for the ${ }^{76} \mathrm{Ge} /{ }^{136} \mathrm{Xe}$ NME has been used.

For each of these cases, we vary $m_{\nu_{1}}, \theta_{L R}$, and $\delta_{C P}$ to obtain the predicted value of the half-life of the $0 \nu \beta \beta$ decay process. Figure 4 shows the variation of $T_{1 / 2}^{0 \nu}$ for the ${ }^{76} \mathrm{Ge}$ nucleus as a function of the lightest neutrino mass $m_{\nu_{1}}$ for fixed values of $\theta_{L R}$ and $\delta_{C P}$. The values of all other PMNS matrix elements were fixed to their central values, and the $\lambda_{R}^{\prime}$ matrix was chosen such that the right-handed neutrino masses were $7.92 \mathrm{eV}, 3.54 \mathrm{keV}$, and $3.55 \mathrm{keV}$, respectively. As can be seen here, the half-life decreases quite drastically as the lightest neutrino mass increases. This is because the light-heavy neutrino mixing increases as discussed earlier, and as a result, the $\eta_{3}, \eta_{\lambda}$, and $\eta_{\eta}$ contributions become dominant. As for this figure, we consider a large value of $\theta_{L R}$; therefore, $\eta_{\eta}$ always dominate. We find that the canonical light neutrino contribution $\eta_{1}$ is rather subdominant in this figure.

Figure 5 shows the variation of $T_{1 / 2}^{0 \nu}$ with the left-right charged gauge boson mixing $\theta_{L R}$. As $\theta_{L R}$ increases, the decay half-life falls drastically for a value $\theta_{L R} \gtrsim 10^{-6}$. This is the point at which the $\eta_{\eta}$ term, which is proportional to $\tan \theta_{L R}$, starts dominating over the other terms resulting in a steep decrease of the half-life as expected. For smaller values of $\theta_{L R}$, the dominant contribution arises from $\eta_{1}, \eta_{3}, \eta_{\lambda}$, which are independent of $\theta_{L R}$, and hence, the curves remain almost horizontal in this region. Since the $C P$ violating phase $\delta_{C P}$ is another crucial parameter in our analysis, we show the variation of half-life with respect to $\delta_{C P}$. Figure 6 gives the change of $T_{1 / 2}^{0 \nu}$ for ${ }^{76} \mathrm{Ge}$ nucleus as a function of $\delta_{C P}$. A close inspection of the numbers we obtained shows that the variation of half-life mirrors the variation in $\sin \delta_{C P}$ which directly determines the values of the neutrino parameters obtained in our calculations. This is to note that, in all these figures, the scenario $\mathrm{H}_{\min }-\mathrm{Ge}_{\max }$ gives the strongest constraint. This can be understood from the expression of $T_{1 / 2}^{0 \nu}$ as given in Eq. (24). As can be seen, 

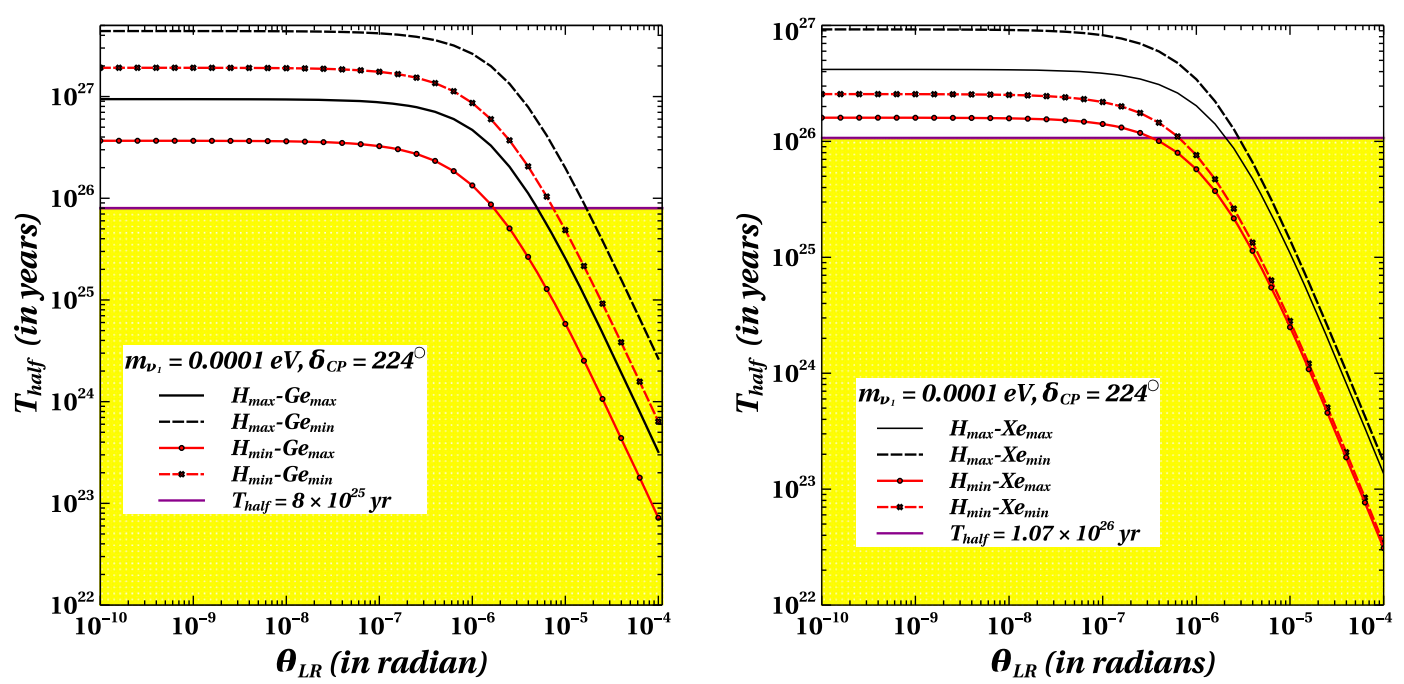

FIG. 5. Half-life of $0 \nu \beta \beta$ process for ${ }^{76} \mathrm{Ge}$ (left) and ${ }^{136} \mathrm{Xe}$ nucleus (right) as a function of left-right charged gauge boson mixing.

the calculated half-life is smaller (leading to a more constrained scenario) for larger values of the amplitudes and NMEs. Therefore, naturally, the maximum values of ${ }^{76} \mathrm{Ge}$ NMEs lead to more constrained scenarios.

The charged Higgs mixing on the other hand plays an indirect but significant role in determining the values obtained for the Feynman diagram amplitudes. As was discussed earlier, the Feynman amplitudes corresponding to $\eta_{2}$, and $\eta_{4}$ are negligible due to the $\left(M_{W_{L}} / M_{W_{R}}\right)^{4}$ suppression. So the dominant contribution always arises from any one of the $\eta_{1}, \eta_{\lambda}$, or $\eta_{\eta}$ terms, while we find that the $\eta_{3}$ contribution is slightly smaller than these above-mentioned contributions. A smaller charged Higgs mixing will invariably lead to a lighter Majorana mass for the right-handed (RH) neutrinos, which has a two-fold effect on the neutrino sector. First, lighter $\mathrm{RH}$ neutrinos will result in relatively heavier active neutrinos since the active neutrino mass is obtained by the seesaw mechanism in our case. This will boost the $\eta_{1}$ amplitude resulting in a smaller value of $T_{1 / 2}$. Second, a heavier active neutrino will result in a larger light-heavy mixing as discussed earlier. This again helps boost the $\eta_{\lambda}$ and $\eta_{\eta}$ amplitudes further lowering the calculated half-life of the $0 \nu \beta \beta$ process, leading to a tight constraint on the parameter.

The plots obtained for the ${ }^{136} \mathrm{Xe}$ nucleus are very similar in nature to the ones for ${ }^{76} \mathrm{Ge}$, and the most constrained scenario is again the $\mathrm{H}_{\min }-\mathrm{Xe}_{\max }$ case. This warrants for a more detailed study of this particular case for a better understanding, which we discuss below. We present the results for both ${ }^{76} \mathrm{Ge}$ and ${ }^{136} \mathrm{Xe}$ nuclei in the ensuing discussion of the most constrained scenario for each, i.e., largest values for the NMEs and minimal mixing of the Higgs sector $\mathrm{H}_{\min }-\mathrm{Ge}_{\max }$ and $\mathrm{H}_{\min }-\mathrm{Xe}_{\max }$.

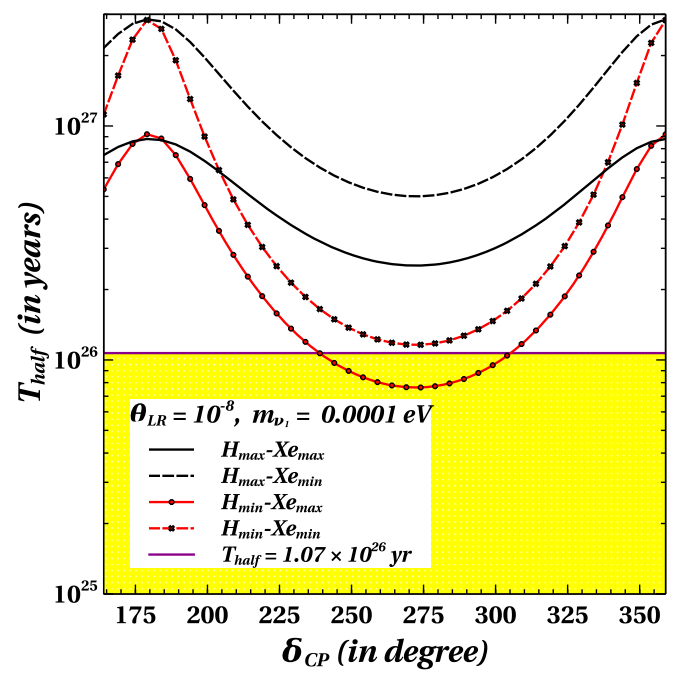

FIG. 6. Half-life of the $0 \nu \beta \beta$ process for ${ }^{76} \mathrm{Ge}$ and ${ }^{136} \mathrm{Xe}$ nuclei as a function of the $C P$ violating phase in the PMNS matrix. 


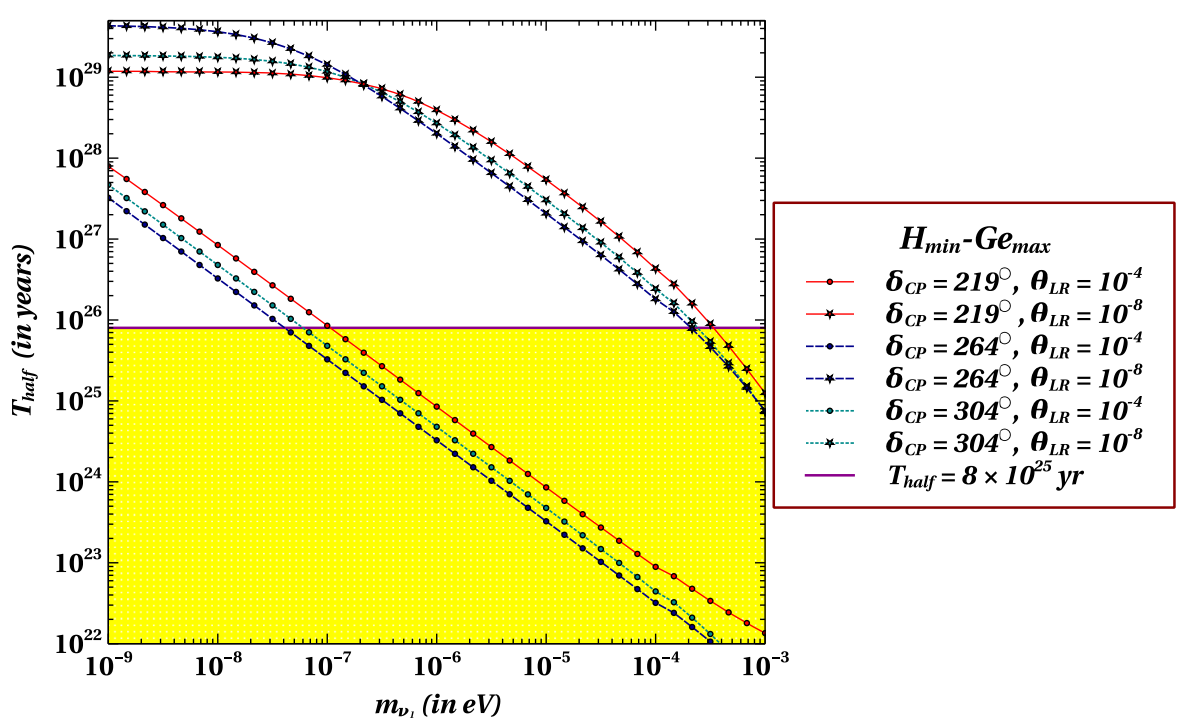

FIG. 7. Half-life of the $0 \nu \beta \beta$ process for the ${ }^{76} \mathrm{Ge}$ nucleus as a function of the lightest neutrino mass for several fixed values of $\theta_{L R}$ and $\delta_{C P}$.

Figure 7 shows the variation of $T_{1 / 2}^{0 \nu}$ for ${ }^{76} \mathrm{Ge}$ as a function of the lightest neutrino mass for several fixed values of $\theta_{L R}$ and $\delta_{C P}$. It is quite clear that the value of $T_{1 / 2}^{0 \nu}$ decreases as the lightest neutrino mass and/or value of $\theta_{L R}$ increases. The variation with $m_{\nu_{1}}$ is quite evident from our earlier discussion. For the set of plots with $\theta_{L R}=10^{-8}$, the initial variation with $m_{\nu_{1}}$ is quite modest until $m_{\nu_{1}} \lesssim 10^{-7} \mathrm{eV}$. The major contribution here comes from the $\eta_{1}$ term with the half-life slowly decreasing with an increase in the lightest neutrino mass. At larger values of $m_{\nu_{1}}$, the light-heavy neutrino mixing increases significantly, and the dominant contribution comes from the $\eta_{\lambda}$ term. The effect of increasing $\theta_{L R}$ can be understood as an artifact of an increase in the $\eta_{\eta}$ term which starts contributing quite significantly at $\theta_{L R} \gtrsim 10^{-6}$. Similar characteristics and dependence can be observed in Fig. 8 where we have plotted the variation of $T_{1 / 2}^{0 \nu}$ as a function of $\theta_{L R}$ for fixed values of $m_{\nu_{1}}$ and $\delta_{C P}$. As can be seen here, the value of $T_{1 / 2}^{0 \nu}$ remains almost constant in the initial region of $\theta_{L R} \lesssim 10^{-6}$. In this region, the dominant contribution comes from $\eta_{1}$ and $\eta_{\lambda}$, and since the lightest neutrino mass is constant for each line, there is no variation in their value. In the region $\theta_{L R} \gtrsim 10^{-6}$, the $\eta_{\eta}$ term starts dominating, and we see a sharp decrease in the value of $T_{1 / 2}^{0 \nu}$ in this region.

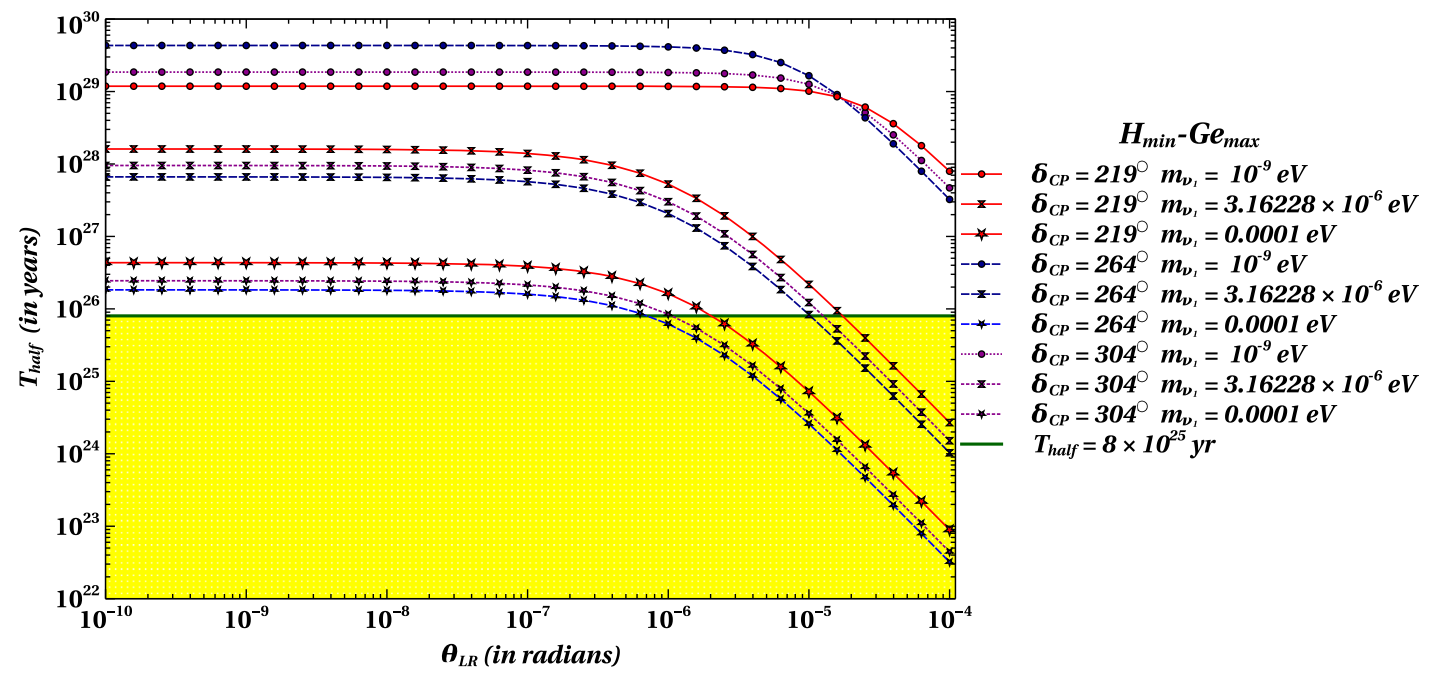

FIG. 8. Half-life of the $0 \nu \beta \beta$ process for the ${ }^{76} \mathrm{Ge}$ nucleus as a function of left-right charged gauge boson mixing for several fixed values of $m_{\nu_{1}}$ and $\delta_{C P}$. 


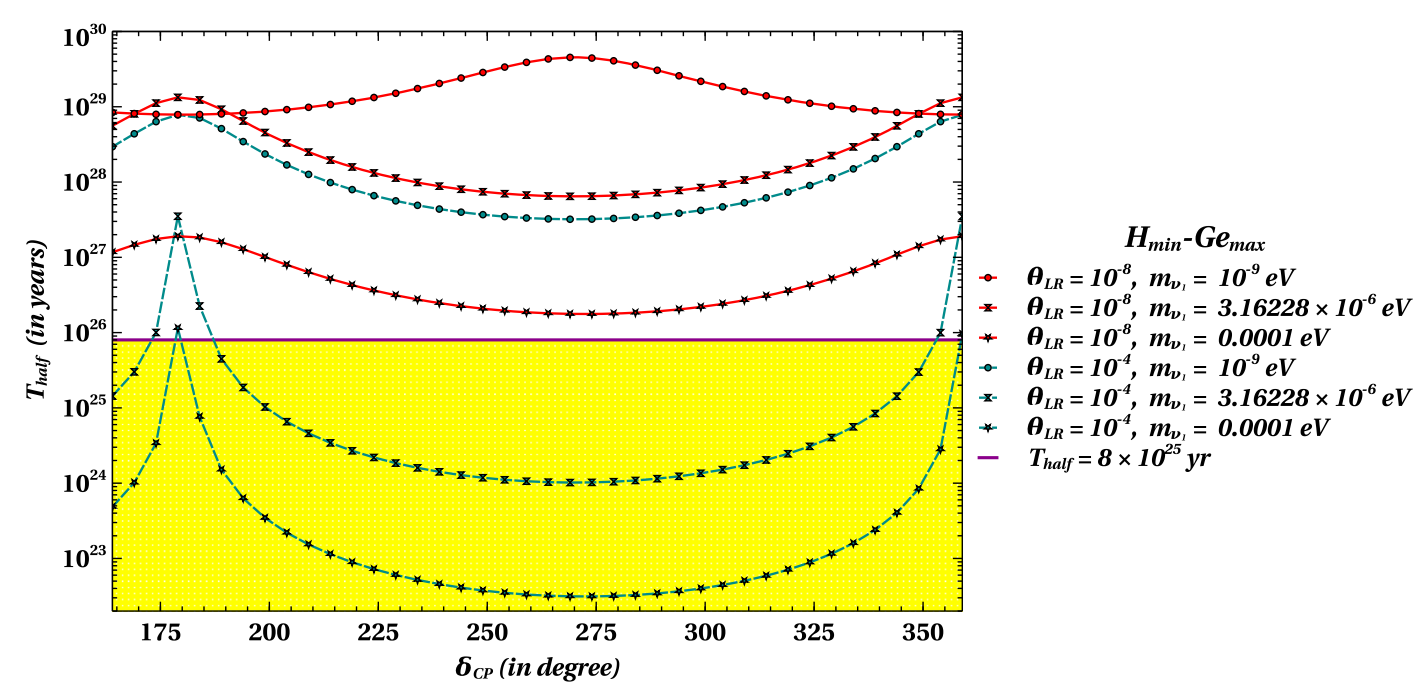

FIG. 9. Half-life of the $0 \nu \beta \beta$ process for the ${ }^{76} \mathrm{Ge}$ nucleus as a function of the $C P$ violating phase of the PMNS matrix for several fixed values of $\theta_{L R}$ and $m_{\nu_{1}}$.
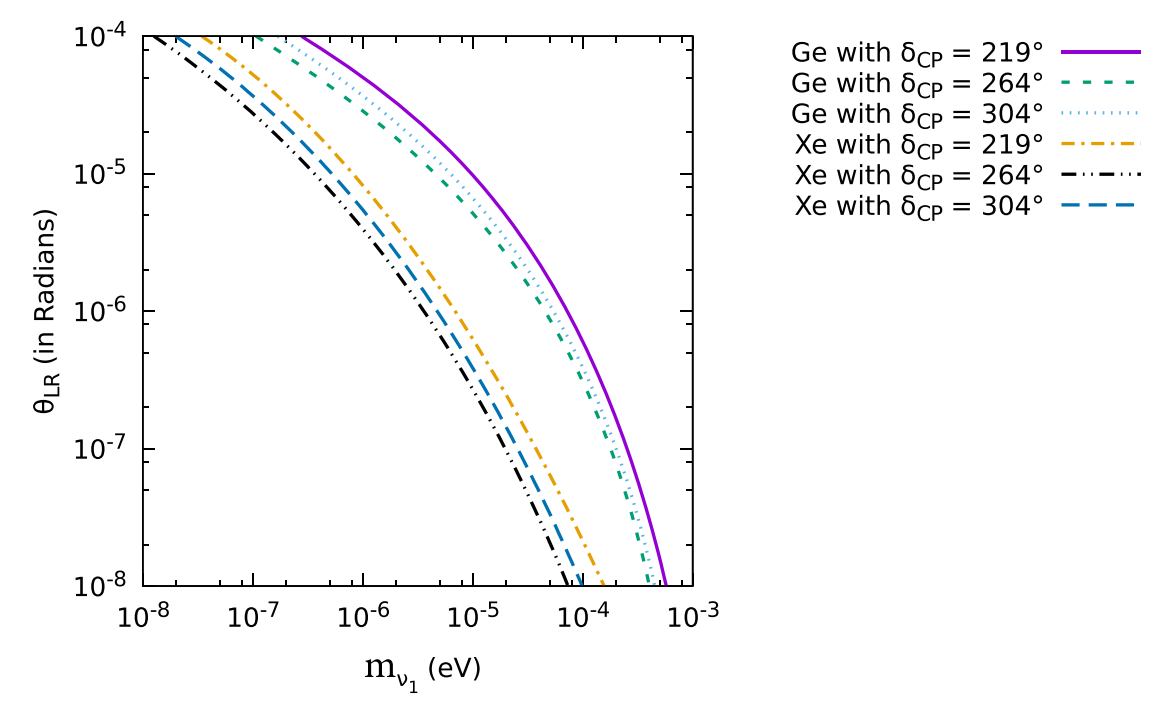

FIG. 10. Limits on the lightest neutrino mass and left-right $W$ gauge boson mixing.

Finally, the variation of $T_{1 / 2}^{0 \nu}$ with $\delta_{C P}$ is given in Fig. 9. This plot is quite interesting as it clearly shows the contribution of different $\eta$ terms in different regions of parameter space. The line corresponding to $\theta_{L R}=10^{-8}$ and $m_{\nu_{1}}=10^{-9} \mathrm{eV}$ has a dominant contribution from the $\eta_{1}$ term. As $\eta_{1}=\frac{1}{m_{e}} \sum_{i} \mathbb{U}_{e i}^{2} m_{i}$, its contribution in this case only depends on the matrix elements $\mathbb{U}_{e i}$ since $m_{e}$ and $m_{i}$ remain constant. The $\mathbb{U}_{13}$ element ${ }^{4}$ is directly proportional to $e^{-i \delta_{C P}}$, and as a result, one can approximately write $T_{1 / 2}^{0 \nu} \sim \frac{1}{G_{01}^{0 \nu}\left|M_{\nu}^{0 \nu} \eta_{1}\right|^{2}}$. As $\delta_{C P}$ approaches $180^{\circ}$ or $360^{\circ}$, the value of $\eta_{1}$ goes through a maxima, while it becomes a minima at

\footnotetext{
${ }^{4}$ The $\mathbb{U}_{13}$ element will be the same as the $(1,3)$ element of the PMNS matrix.
}

$\delta_{C P}=270^{\circ}$. The inverse of this behavior is reflected in the $T_{1 / 2}^{0 \nu}$ plot. For the other lines in this figure, they receive dominant contributions from either $\eta_{\lambda}$ or $\eta_{\eta}$. Here, as $\mathbb{U}_{13}$ decreases, the elements of $\mathbb{S}$ and $\mathbb{T}$ mixing matrices increase, and hence, their nature is opposite to the previous plot. The natures of the plots for the ${ }^{136} \mathrm{Xe}$ nucleus are the same as the ${ }^{76} \mathrm{Ge}$ nucleus except for the fact that the parameters are more tightly constrained owing to the more stringent experimental limit of the $0 \nu \beta \beta$ half-life for the ${ }^{136}$ Xe nucleus.

Figure 10 shows the upper limits on the mass of the lightest neutrino in the this model as a function of $\theta_{L R}$ for fixed values of $\delta_{C P}$ for both ${ }^{76} \mathrm{Ge}$ and ${ }^{136} \mathrm{Xe}$ nuclei. As we have already discussed that the most stringent bound on the parameter space is obtained for a $\delta_{C P}$ of around $264^{\circ}$; this 
fact is also reflected from this figure. As expected from the previous discussion, the upper limit on the lightest neutrino mass becomes much stronger for larger values of $\theta_{L R}$ and vice versa. This occurs due to a dominant $\eta_{\eta}$ contribution for a large $\theta_{L R}$, leading to a tighter constraint on the lightest neutrino mass $m_{\nu_{1}}$. Another observation is that the limits obtained for the ${ }^{136} \mathrm{Xe}$ nucleus are much stronger than for the ${ }^{76} \mathrm{Ge}$ nucleus.

\section{EXTENDED SEESAW MODEL AND ANALYSIS}

This is another extension of SM, where the model contains light and heavy sterile neutrinos, ${ }^{5}$ which can give large contributions in the $0 \nu \beta \beta$ process. Several studies $[43,44,60,62,82-96]$ have focused on sterile neutrinos with masses $>100 \mathrm{MeV}$ and large contributions in the lepton number violating process. Here, instead, we consider some of the sterile neutrino states in the $<100 \mathrm{MeV}$ mass range. We investigate the allowed model parameters, which satisfy a number of theoretical and experimental constraints. In doing so, we first consider a simplistic one-generation scenario with one active neutrino, one light sterile neutrino $S_{L}$, and another heavy sterile neutrino $N_{L}$. Subsequently, we extend our analysis with a realistic three-generation case where the neutrino sector comprises three active neutrinos along with an additional six sterile neutrinos (three $S_{L}$ and three $N_{L}$ ) which are neutral under the SM gauge group. Below, we first review the model and then discuss the contribution in the $0 \nu \beta \beta$ process.

\section{A. Model}

The neutral lepton sector of the model contains three generations of SM neutrinos $\nu_{L}$ along with additional sterile neutrino states $S_{L}$ and $N_{L}$. The mass terms of the neutrinos have the following form:

$$
\begin{aligned}
\mathcal{L}= & -\frac{1}{2}\left(\begin{array}{lll}
\nu_{L} & S_{L} & N_{L}
\end{array}\right)\left(\begin{array}{ccc}
0 & 0 & M_{D}^{T} \\
0 & \mu & M_{S}^{T} \\
M_{D} & M_{S} & M_{R}
\end{array}\right)\left(\begin{array}{c}
\nu_{L} \\
S_{L} \\
N_{L}
\end{array}\right) \\
& + \text { H.c.. }
\end{aligned}
$$

We denote the neutral lepton mass matrix as $\mathcal{M}_{n}$, and hence,

$$
\mathcal{M}_{n}=\left(\begin{array}{ccc}
0 & 0 & M_{D}^{T} \\
0 & \mu & M_{S}^{T} \\
M_{D} & M_{S} & M_{R}
\end{array}\right) \text {. }
$$

We choose to work in a basis where the Majorana mass matrix $M_{R}$ of $N_{L}$ sterile is real. The term containing $\mu$ denotes the Majorana mass of the heavy neutrino state $S_{L}$ with $\mu$ being a complex symmetric matrix. The Dirac mass matrix $M_{D}$ represents the mixing between the SM neutrino states $\nu_{L}$ and the heavy sterile neutrino states $N_{L}$, whereas $M_{S}$ represents the mixing between the two sterile states $S_{L}$ and $N_{L}$. Throughout our analysis, we consider that the matrices $M_{R}$ and $M_{S}$ are invertible. We also assume that the different submatrices follow the hierarchy $M_{R}>M_{S}>$ $M_{D} \gg \mu$. For the seesaw approximation to be valid, the mixing matrices should satisfy $\mu<M_{S}^{T} M_{R}^{-1} M_{S}$, i.e., $\mu<\mathcal{O}\left(\frac{M_{S}^{2}}{M_{R}}\right)$, see [15] for details.

Contrary to the inverse seesaw [97-108], the extended seesaw model contains both the heavy and small lepton number violation scales $M_{R}$ and $\mu$, respectively. The SM neutrino masses strongly depend on the small lepton number violating scale $\mu$, and hence, in the $\mu \rightarrow 0$ limit, the $\nu_{L}$ states become massless. The heavy Majorana neutrino contribution in $0 \nu \beta \beta$ decay can be sizeable, even in the $\mu \rightarrow 0$ limit. Hence, the contributions of the SM neutrinos and the heavy Majorana neutrinos in the $0 \nu \beta \beta$ process are completely decoupled from each other. The contribution from heavy sterile neutrinos for this model has been discussed in [15].

The neutrino mass matrix $\mathcal{M}_{n}$ can be diagonalized by a unitary transformation,

$$
\mathcal{U}^{T} \mathcal{M}_{n} \mathcal{U}=\mathcal{M}_{n}^{d}
$$

where $\mathcal{U}$ as an expansion with order parameter $M_{D} / M_{S}$ has the following form [15]:

$$
\left(\begin{array}{ccc}
\left(1-\frac{1}{2} M_{D}^{\dagger}\left(M_{S}^{-1}\right)^{\dagger} M_{S}^{-1} M_{D}\right) W_{\mu} & M_{D}^{\dagger}\left(M_{S}^{-1}\right)^{\dagger} W_{S} & M_{D}^{\dagger} M_{R}^{-1} W_{N} \\
-M_{S}^{-1} M_{D} W_{\mu} & \left(1-\frac{1}{2} M_{S}^{-1} M_{D} M_{D}^{\dagger}\left(M_{S}^{-1}\right)^{\dagger}-\frac{1}{2} M_{S}^{\dagger} M_{R}^{-2} M_{S}\right) W_{S} & M_{S}^{\dagger} M_{R}^{-1} W_{N} \\
M_{S}^{T-1} \mu M_{S}^{-1} M_{D} W_{\mu} & -M_{R}^{-1} M_{S} W_{S} & \left(1-\frac{1}{2} M_{R}^{-1} M_{S} M_{S}^{\dagger} M_{R}^{-1}\right) W_{N}
\end{array}\right) .
$$

\footnotetext{
${ }^{5}$ We denote the gauge singlet neutrinos as sterile neutrinos, as they are not charged under the SM gauge group.
} 
In the above, $W_{\mu}, W_{S}$, and $W_{N}$ are the three unitary matrices that diagonalize the block diagonal matrices

$$
\begin{aligned}
& m_{\nu} \sim M_{D}^{T}\left(M_{S}^{-1}\right)^{T} \mu\left(M_{S}\right)^{-1} M_{D}, \quad m_{s} \sim-M_{S}^{T}\left(M_{R}\right)^{-1} M_{S} \\
& m_{n} \sim M_{R} .
\end{aligned}
$$

The matrix $m_{\nu}$ represents the light neutrino mass matrix, and $m_{s}$ and $m_{n}$ represent the heavy neutrino mass matrices. The hierarchy among the submatrices ensures that $m_{n}$ and its eigenvalues give the heaviest sterile neutrinos in this model. The other sterile neutrinos that originate from the diagonalization of $m_{s}$ can be relatively lighter, but they certainly should be heavier than the three active neutrinos $m_{\nu}<m_{s}<m_{n}$. In the subsequent sections, we explore the possibility of that the sterile states from $m_{s}$ are in the $\mathrm{eV}$ to $\mathrm{MeV}$ range, while the remaining sterile neutrino states $m_{n}$ are more than $\mathrm{GeV}$. Before presenting a detailed analysis on $0 \nu \beta \beta$, we first consider additional constraints coming from light neutrino mass measurement, nonunitarity. and others.

\section{B. Constraints}

Before delving into the analysis, we present short descriptions of all constraints that have been applied in this model.

\section{(a) Theoretical Constraints:}

Hierarchy: The different submatrices of Eq. (26) should satisfy the hierarchy

(i) $M_{R}>M_{S}>M_{D} \gg \mu$, and

(ii) $M_{S}^{T} M_{R}^{-1} M_{S}>\mu$; i.e., $m_{s}>\mu$ [from Eq. (30)]. For one generation, this will be $M_{S}^{2} / M_{R}>\mu$. This limit also defines the region where the seesaw approximation is valid [15].

Unitarity: With the mass matrix being symmetric, the diagonalization matrix given in Eq. (28) should be orthogonal or unitary. That is, we should have the relation $\mathcal{U}^{\dagger} \mathcal{U}=\mathcal{U U}^{\dagger}=\mathbf{I}$. But working with the seesaw approximation up to a certain order and also having low-scale sterile, the elements of $U=\mathcal{U U}^{\dagger}$ will not be an identity matrix, rather those elements will be $\mathbf{I} \pm \delta$, where $\delta$ is the tolerance of every single elements of $U$ to get a viable parameter space for lightest sterile neutrino ${ }^{6}$ in this model. So, to zero in on the allowed parameter space of $\mathrm{eV}$ to $\mathrm{MeV}$ sterile in this model, we have to constrain the parameter space, setting some cutoff values on the both diagonal and nondiagonal elements of $U$. In short, we allow some error bars on the diagonal elements on $U$ around unity, and for nondiagonal elements, the required error bar will be around zero. Depending on the choice of parameter space, the error bar may differ for diagonal and nondiagonal elements. We have generally taken

\footnotetext{
${ }^{6}$ See Sec. III D (Analysis section).
}

the maximum constraints on the deviation which provide us the desired allowed parameter space.

(b) Experimental Constraint:

Mass of active neutrino: We consider the constraint on the sum of active neutrino masses from Planck cosmological data [49], i.e., at 95\% C.L., the sum of the masses of active neutrinos will be less than $\sum m_{\nu}<0.194 \mathrm{eV}$. In the analysis of one generation, this bound simply manifests as the upper bound on the mass of single active neutrino. We implement the constraints on mixing angles and on the mass-square differences among three active neutrinos from neutrino oscillation data in the three-generation case $[1,109]$ in the case of normal hierarchy.

Constraints from $0 \nu \beta \beta$ limit: The limit on $T_{1 / 2}^{0 \nu}$ from the KamLAND-Zen [46] severely constrains the parameter space for $\mathrm{eV} / \mathrm{MeV}$ sterile neutrino of this model, see Sec. III C.

Daya Bay experiment: The Daya Bay reactor antineutrino experiment shows a large exclusion region between $2 \times 10^{-4}<\Delta m_{s 1}^{2}<0.3 \mathrm{eV}^{2}$ as a function of $\sin ^{2} 2 \theta_{1 s}[110]$ at $95 \%$ C.L., where $\Delta m_{s 1}^{2}$ is the mass-square difference between extra sterile and electron neutrinos $\left(\nu_{e}\right)$, and $\theta_{1 s}$ is the angle of active-sterile mixing. This result will further constrain the allowed parameter space for $\mathrm{eV}$ sterile neutrinos.

\section{C. $0 \nu \beta \beta$ decay: Sterile neutrino contributions}

In this section, we outline the contributions of sterile neutrinos having Majorana masses in $0 \nu \beta \beta$ decay.

The half-life of $0 \nu \beta \beta$ is written as $[82,111-113]$

$$
\frac{1}{T_{1 / 2}^{0 \nu}}=K_{0 \nu}\left|\Theta_{e j}^{2} \frac{\mu_{j}}{\left\langle p^{2}\right\rangle-\mu_{j}^{2}}\right|^{2},
$$

where $j$ represents the number of light neutrino states and the additional heavy neutrino states. The parameters $\mu_{j}$ and $\Theta_{e j}$ represent the masses of the neutrino states and the mixing with SM neutrinos, respectively. In the above, $K_{0 \nu}=G_{0 \nu}\left(\mathcal{M}_{N} m_{p}\right)^{2}$, and $\left\langle p^{2}\right\rangle \equiv-m_{e} m_{p} \frac{\mathcal{M}_{N}}{\mathcal{M}_{\nu}}$. The reference mass scales are considered as electron $\left(m_{e}\right)$ and proton $\left(m_{p}\right)$ masses, and $M_{\nu}$ and $M_{N}$ are the NMEs for exchange of light and heavy neutrinos, respectively. The values of NME and the phase space factor $G_{0 \nu}$ have been taken from Ref. [114]. Below, we classify the sterile neutrino contributions according to their mass scale.

Other than the contributions of the SM neutrinos, the sterile neutrino states $S_{k}$ and $N_{k}(k=1,2,3$; in our case) can also contribute in the $0 \nu \beta \beta$ process. Evidently, we have two extra contributions apart from the SM one.

(a) The heaviest states $N_{k}$ have a mass range $m_{n_{k}}>$ $100-200 \mathrm{MeV}$ and give a contribution in $0 \nu \beta \beta$ as 


$$
A_{N} \sim \frac{V_{e N_{k}}^{2}}{m_{n_{k}}},
$$

where $A_{N}$ represents the amplitude of this process, and $V_{e N_{k}}$ is the mixing of the $N_{k}$ states with the active neutrinos. Using $V_{e N}=M_{D}^{\dagger} M_{R}^{-1} W_{N}$, this can be simplified from the matrix form of $A_{N}$ as

$$
\begin{aligned}
A_{N} & \sim V_{e N_{k}} M_{R}^{-1} V_{e N_{k}}^{T} \\
& \sim M_{D}^{T} M_{R}^{-1} W_{N} M_{R}^{-1} W_{N}^{T}\left(M_{R}^{-1}\right)^{T} M_{D} .
\end{aligned}
$$

Since, $M_{R}$ is chosen to be diagonal and $W_{N}$ being the corresponding diagonalization matrix, $A_{N}$ can be simplified as

$$
A_{N} \sim\left(M_{D}^{T} M_{R}^{-3} M_{D}\right)_{e e},
$$

as $W_{N}$ is the unit matrix in our scenario.

(b) The other sterile neutrino states $S_{k}$ give contributions proportional to

$$
A_{S} \sim \frac{V_{e S_{k}}^{2}}{m_{s_{k}}}
$$

for the mass range $m_{s_{k}}>100-200 \mathrm{MeV}$, whereas

$$
A_{S} \sim \frac{V_{e S_{k}}^{2} m_{s_{k}}}{\left\langle p^{2}\right\rangle}
$$

when sterile mass is light. We use the compact expression for the amplitude that also takes into account $\left\langle p^{2}\right\rangle \simeq m_{s_{k}}^{2} \simeq 100 \mathrm{MeV}^{2}$,

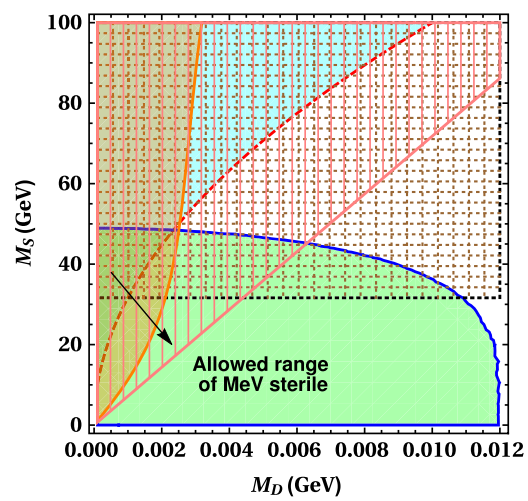

(a)

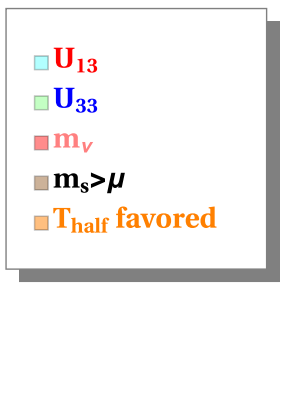

$\square \mathbf{m}_{\mathrm{s}}>\boldsymbol{\mu}$

$\square T_{\text {half }}$ favored

FIG. 11. Allowed parameter space of a light sterile neutrino in (a) $\mathrm{MeV}$ and in (b) $\mathrm{eV}$ ranges as a function of the model parameters $M_{D}$ and $M_{S}$ in the extended seesaw scheme. The regions have been obtained from theoretical constraints, light neutrino measurements, and $0 \nu \beta \beta$ results. The parameter $\mu$ has been set to $10^{-2}$ and $10^{-10} \mathrm{GeV}$ for $\mathrm{MeV}$ and eV ranges, respectively. In both cases, $M_{R}=10^{5} \mathrm{GeV}$. The orange-shaded region of (b) will be constrained further from the Daya Bay reactor antineutrino experiment [110] that is elaborated in Fig. 12. 
one-generation scenario. The matrix $\mathcal{M}_{n}$ in Eq. (27) is $3 \times 3$ instead of being $9 \times 9$ in this case. The square boxes in the index box of this figure [Fig. 11(a)] shows the color of allowed regions in agreement with different constraints, and the respective texts are written in the same color as the border of that region. The cyan-colored region enclosed by the red-dashed curve in Fig. 11(a) shows the region allowed by the off-diagonal element $U_{13}$, where $U_{13}$ is the (1,3) element of $\mathcal{U}^{\dagger} \mathcal{U}$ with $\mathcal{U}$ being the diagonalization matrix [Eq. (28)]. Here, we consider the constraint as $\left|U_{13}\right|<10^{-8}$; i.e., $U_{13}$ is almost vanishing. The lower green region enclosed by the blue solid line presents the region allowed by the diagonal element $U_{33}$. The constraints due to other matrix elements of $U$ coming from the condition of diagonalization matrix $\mathcal{U}$ being unitary are less stringent and are simply allowed by the final overlapped zone. In that final allowed region, the numerical values of the offdiagonal element $U_{13}$ is $\sim 10^{-9}$ and that of the diagonal element $U_{33}$ are $\sim\left(1+10^{-16}\right)$. The region covered by pinkcolored straight lines shows the mass of the light neutrino range $0<m_{\nu}<0.194 \mathrm{eV}$. The brown rectangle region enclosed from below by the black dashed line (near $M_{S}=32 \mathrm{GeV}$ ) represents the constraint $m_{s}=M_{S}^{2} / M_{R}>\mu$ marking the area where the seesaw approximation is valid. The extreme left almost vertical orange-colored region enclosed by the solid orange bow-type curve shows the region in agreement with the contribution of $0 \nu \beta \beta$, where $T_{1 / 2}^{0 \nu}>1.07 \times 10^{26} \mathrm{yr}$ [46]. In obtaining this allowed parameter space, we have considered both the light neutrino and sterile neutrino contributions, see Eq. (38). The values of the NMEs that we have considered in this analysis are $M_{\nu}=2.29$ and $M_{N}=163.5$ [114].

The overlapped region in Fig. 11(a) enclosed by a dashed black straight line from below and solid blue line from above with $32 \mathrm{GeV}<M_{S}<49 \mathrm{GeV}$ and red-dashed line from the right is the final allowed range for the $\mathrm{MeV}$ sterile neutrino in the extended seesaw model, with the value of $M_{D} \leq 0.00011 \mathrm{GeV}$ for $M_{S} \sim 32 \mathrm{GeV}$ and with the $M_{D}$ value up to $\sim 0.002356 \mathrm{GeV}$ for $M_{S}=49 \mathrm{GeV}$. The allowed mass range of $m_{s}$ is $10 \mathrm{MeV}<m_{s}<24 \mathrm{MeV}$. The mass of the active neutrino in that region is $m_{\nu} \leq 10^{-2} \mathrm{eV}$.

The plot in the right panel [Fig. 11(b)] of Fig. 11 shows the allowed region for the $\mathrm{eV}$ sterile neutrino in the extended seesaw model and its contribution to $0 \nu \beta \beta$ decay in the $M_{D}-M_{S}$ plane. The inclusion of sterile neutrinos whether being heavy or light has its effect on the unitarity of the PMNS matrix [50]. The PMNS matrix encoding the nonunitarity effect due to the mixings of active-sterile neutrinos is given by [50]

$$
\mathcal{N}=(I-\alpha) \mathcal{U}^{\prime}
$$

where, $\mathcal{U}^{\prime}$ is equivalent to a standard PMNS matrix which is also a unitary matrix having a small deviation proportional $\alpha$. Clearly, from Eq. (29), we can see that $\alpha$ being theoretically the same for all elements is given by $1 / 2\left(M_{D}^{2} / M_{S}^{2}\right)$ in the extended seesaw model, whereas $\mathcal{U}^{\prime}$ is equivalent to $W_{\mu}$ of the same equation (29). The general form of mixing terms is usually given by the ratio between the mass scales of light neutrinos and the sterile neutrinos. Therefore, in the case of light sterile neutrinos $(\sim \mathrm{eV})$, with the active neutrino and the light sterile mass scales being very close, the effect of mixing cannot be ignored. So the light sterile neutrino has a strong impact on the deviation of the PMNS matrix from being unitary. In Ref. [50], the constraint on $\alpha$ is given for different mass values of sterile neutrinos. For the mass-square difference of sterile and active neutrinos in the $\mathrm{eV}^{2}$ regime, at $95 \%$ C.L., the bound is given by $\alpha<10^{-2}$. So, in the case of $\mathcal{N}^{\dagger} \mathcal{N}$ and for one generation, this bound is manifested as of the order of $\sim 10^{-4}$ as the deviation from unity (for the element $U_{11}$ ).

Therefore, in Fig. 11(b), the constraint on $U_{11}$ is taken as $\left|U_{11}-1\right|<10^{-4}$. Since, the matrix $\mathcal{U}_{22}$ in Eq. (29) also contains a term like $\left[1-1 / 2\left(M_{D}^{2} / M_{S}^{2}\right)-1 / 2\left(M_{S}^{2} / M_{R}^{2}\right)\right]$ with $M_{R}$ being heavy, this term can be effectively written as $\left[1-1 / 2\left(M_{D}^{2} / M_{S}^{2}\right)\right]$; so the constraint on $U_{22}$ will be the same as that of $U_{11}$. The cyan-colored region surrounded by the red-dashed curve shows the allowed region for an $\mathrm{eV}$ sterile neutrino from the limit applied on $U_{11}$. The pinkcolored region shows the allowed region for $\mathrm{eV}$ sterile neutrinos as $\left|U_{13}\right|<10^{-8}$. The brown-colored region shows the corresponding allowed range in agreement with the hierarchy of the model parameters $M_{S}>M_{D}$. The black-colored region depicts the allowed region from $0 \nu \beta \beta$ decay [46] where contributions of $\mathrm{eV}$ sterile and active neutrinos have been considered. The green-colored region of the oblique line shows the validity range of the seesaw approximation. The final allowed region is given by the orange + yellow-colored shaded region. The allowed region is enclosed by a green line from below (at $M_{S}=0.0032 \mathrm{GeV}, M_{D} \sim 5 \times 10^{-4}$ to $0.002 \mathrm{GeV}$ ), by a tilted red-dashed line from the lower right $\left[\left(M_{D}, M_{S}\right) \sim 5 \times 10^{-4}, 0.0032 \mathrm{GeV}\right.$ up to $\left(M_{D}, M_{S}\right) \sim$ $(0.002,0.02) \mathrm{GeV}]$, and finally by an almost vertical black line from the right side. The region can be further extended leftwards and upwards by lowering the value of $M_{D}$ and increasing the value of $M_{S}$, respectively. The other constraints such as unitarity constraints from other elements of $U$ and limits from neutrino masses are allowed by the final overlapped region. The lowest allowed value of model parameter $M_{S}$ is $0.0032 \mathrm{GeV}$ at $M_{D}=10^{-5} \mathrm{GeV}$. Evidently, the lowest values of sterile neutrinos from the allowed region showed in this figure are about $0.1024 \mathrm{eV}$ and $m_{\nu} \leq 10^{-3} \mathrm{eV}$. In this region, the $U_{11} \sim U_{22} \sim 1+$ $10^{-7}$ approximately, and $U_{13} \sim 10^{-9}$. The values of NMEs are the same as those in Fig. 11(a).

Since, in the extended seesaw, we have eV sterile neutrinos starting from $\sim 0.1 \mathrm{eV}$, and the region can further be constrained from reactor antineutrino experiments, such 


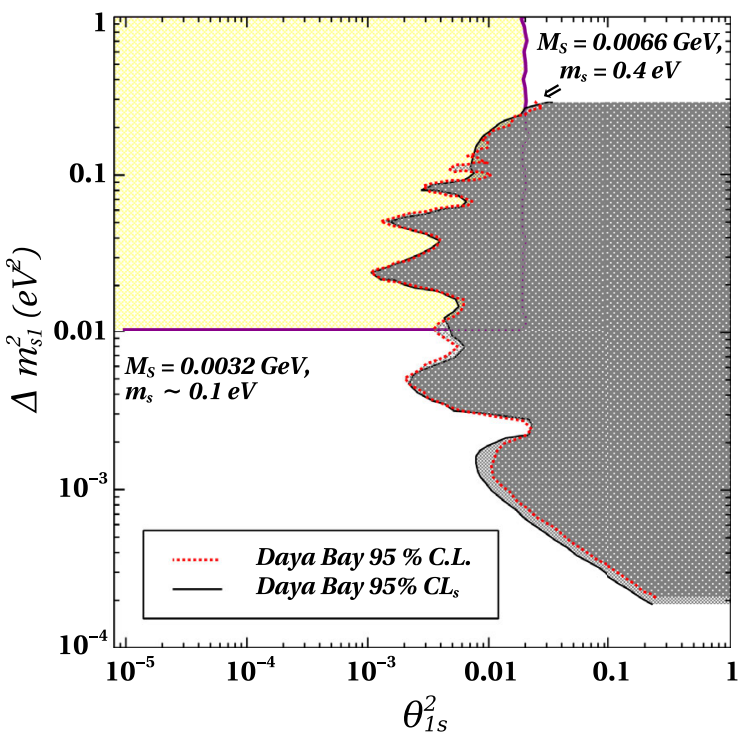

FIG. 12. Constraining the region of eV sterile neutrinos from the Daya Bay reactor neutrino experiment. The gray-colored region from the right side shows the exclusion region from Daya Bay results, whereas the yellow-colored region shows the zone allowed for $\mathrm{eV}$ sterile neutrinos.

as Daya Bay. Evidently, the orange-shaded region of Fig. 11(b) or a part of it, where we have sterile $\sim \mathcal{O}(\mathrm{eV})$, can be probed again from such results. We represent the mentioned region of Fig. 11(b) of the $M_{S}-M_{D}$ plane in $\Delta m_{s 1}^{2}\left(\mathrm{eV}^{2}\right)-\theta_{1 s}^{2}$ in Fig. 12. The filled in yellow box covered by the magenta color line corresponds to the aforementioned region of eV sterile neutrinos of Fig. 11(b). The lower line corresponds to the value $M_{S}=0.0032 \mathrm{GeV}$, where the upper line corresponds to $M_{S}=0.01 \mathrm{GeV}$.

The region in Fig. 11(b) below the green line and right side of the red-dashed line being completely ruled out from our model parameters [Fig. 11(b)] represents the white regions of Fig. 12. The dotted red and black solid lines represent the Daya Bay experimental constraints on lowscale sterile neutrinos in the $\Delta m_{s 1}^{2}\left(\mathrm{eV}^{2}\right)-\theta_{1 s}^{2}$ plane [110]. The gray-colored region shows the zone that is not allowed by this experimental data. The overlapped region of this adjacent figure is ruled out from the experimental result. We can see that Daya Bay results exclude some region from the left side (giving constraint on $M_{D}$ ) but still allow all the values of sterile neutrino from 0.1 to $0.4 \mathrm{eV}$. The mass of the active neutrino in the remaining allowed zone is $m_{\nu} \leq 10^{-4} \mathrm{eV}$.

In passing, we would like to comment on the activesterile mixing value which is getting constrained from Daya Bay data. The $\theta_{1 s}^{2}$ is actually $\left(M_{D} / M_{S}\right)^{2}$. Also, from unitarity [50], we have $\frac{1}{2}\left(M_{D} / M_{S}\right)^{2}<10^{-2}$. Figure 12 shows for some $M_{S}, M_{D}$ values $\theta_{1 s}^{2}<10^{-3}$ giving slightly more stringent bounds on the mixing compared to that of coming from unitarity in our model setup.

\section{Three generation}

In Sec. III D 1, we have discussed different constraints from neutrino mass, half-life of $0 \nu \beta \beta$ decay, and unitarity and the validation of the seesaw approximation for the one-generation realization of the extended seesaw model. In this section, we are extending the analysis for the threegeneration case which is more realistic than the previous scenario. In addition to the bounds from $0 \nu \beta \beta$ and other experimental and theoretical constraints, we also satisfy neutrino oscillation data. In particular, we consider the following:

(i) upper bound on the sum of all three active neutrinos is constrained from cosmology, $\sum_{i} m_{\nu_{i}}<0.194 \mathrm{eV}$ at $2 \sigma$ C.L. [49],

(ii) two mass-square differences $6.93<\frac{\Delta m_{21}^{2}}{10^{-5}} \mathrm{eV}^{2}<7.97$ and $2.37<\frac{\Delta m_{31}^{2}}{10^{-3}} \mathrm{eV}^{2}<2.63$ vary in the $3 \sigma$ range [1],

(iii) $3 \sigma$ range [1] of the three mixing angles $30^{\circ}<\theta_{12}<36.51^{\circ}, \quad 37.99^{\circ}<\theta_{23}<51.71^{\circ}, \quad$ and $7.82^{\circ}<\theta_{13}<9.02^{\circ}$

In the present setup, contribution to the $0 \nu \beta \beta$ can come from light active neutrinos $\left(m_{\nu_{i}}, i=1,2,3\right)$, additional $\mathrm{eV}$ to $\mathrm{MeV}$ scale sterile neutrinos $\left(m_{s_{i}}, i=1,2,3\right)$, and heavy $\mathrm{GeV}$ scale neutrinos $\left(m_{n_{i}}, i=1,2,3\right)$. As shown in Sec. III B, the contributions of the heaviest sterile neutrinos to $0 \nu \beta \beta$ are evidently suppressed. Here, the neutrino mass matrix is $9 \times 9$, but we are working in the seesaw approximation regime which gives three sets of $3 \times 3$ matrices, namely, $m_{\nu}, m_{s}$, and $m_{n}$. After diagonalization of each $3 \times 3$ block of Eq. (30) individually, we check the unitarity constraints as described in Secs. III B and III D 1. The matrices which diagonalize each blocked matrix combine to form a $9 \times 9$ matrix [Eq. (29)], and we impose constraints on the unit matrix ( $U$, see Sec. III D 1$)$ with the absolute variation of each element by $\pm 10^{-2}$. This $\pm 10^{-2}$ variation manifestly impose constraints on the ratio $M_{D} / M_{S}$. We have checked that the error bar is consistent with the experimental bound [50] that arises due to the nonunitarity effect for $\mathrm{eV}-\mathrm{keV}$ scale sterile neutrinos. A detailed description of different conditions provided in Sec. III B to constrain the parameter space have been thoroughly followed in the present analysis.

As discussed earlier, after using the seesaw approximation, we get three different $3 \times 3$ matrices, which are $m_{n}$, $m_{s}, m_{\nu}$. Among them, one corresponds to the mass matrix for the three active neutrinos (denoted by $m_{\nu}$ ), and other two correspond to the mass matrices for the three relatively light sterile neutrinos (denoted by $m_{s}$ ) and the three heavy neutrinos which are in $\mathrm{GeV}$ scale (denoted by $\left.m_{n}\right)$. From Eq. (30), we can see that the expressions of $m_{\nu}$, $m_{s}$, and $m_{n}$ depend on the matrices $M_{D}, M_{R}, M_{S}$, and $\mu$ whose elements are the free input parameters in the extended seesaw scenario. We choose the model parameters in our framework in a way so that we can accommodate $\mathrm{eV}$ to $\mathrm{MeV}$ scale sterile neutrinos. Before proceeding, we consider a few assumptions which include 

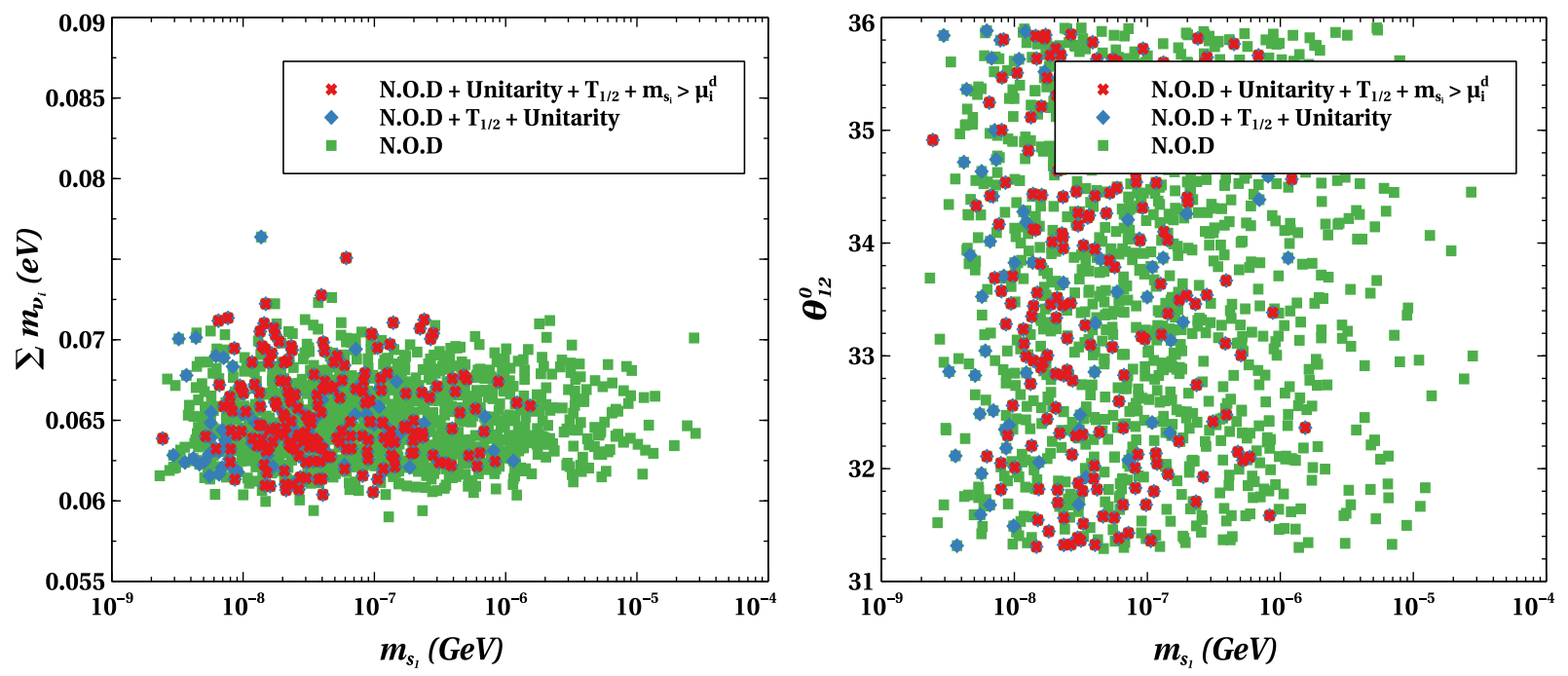

FIG. 13. Scatter plots in $\sum m_{\nu_{i}}(\mathrm{eV})-m_{s_{1}}(\mathrm{GeV})$ (left) and $\theta_{12}-m_{s_{1}}(\mathrm{GeV})$ (right) planes after satisfying the constraints as mentioned in the text. N.O.D represents the constraint from neutrino oscillation data.

$M_{D}, M_{S}, M_{R}$ as the real diagonal matrices and $\mu$ as the complex symmetric matrix, i.e., $\mu^{T}=\mu$ and $\mu^{*} \neq \mu$. In this work, we have focused on the normal hierarchy of the neutrino masses as an illustrative example. In order to satisfy neutrino oscillation constraints and to obtain sterile neutrinos in the $\mathrm{eV}$ to $\mathrm{MeV}$ scale, we have varied the model parameters in the following range (in $\mathrm{GeV}$ ):

$$
\begin{aligned}
10^{-5} & \leq M_{D i i} \leq 10^{-1} \\
10^{-3} & \leq M_{S i i} \leq 10^{-1} \\
50 & \leq M_{R i i} \leq 500 \\
10^{-11} & \leq \mu_{i j}^{R I} \leq 10^{-8}
\end{aligned}
$$

where $i, j$ can vary from 1 to 3 . Moreover, we can choose different ranges of $M_{D}, M_{S}, M_{R}$, and $\mu$ as well which will evade all the theoretical and experimental constraints, but we have considered the heaviest right-handed neutrinos in the three-generation scenario which can be probed in collider experiments. Below, we show the allowed model parameters as well as the correlations among different observables for this model as scatter plots.

In the left panel of Fig. 13, we have shown the allowed region in the $\sum m_{\nu_{i}}(\mathrm{eV})-m_{s_{1}}(\mathrm{GeV})$ plane after satisfying all data, ${ }^{7}$ where $m_{s_{1}}$ is the physical mass of the lightest sterile neutrino state. In the figure, green dots show the range allowed by neutrino oscillation data (N.O.D), and blue rhombus points represent the range allowed by $0 \nu \beta \beta$ and unitarity along with N.O.D. Finally, the red points exhibit the range that is being further constrained by

\footnotetext{
${ }^{7}$ We get similar kinds of behaviors with the other two light sterile neutrinos $s_{2,3}$. Moreover, $s_{2,3}$ also gets similar kinds of masses and contributes to $0 \nu \beta \beta$ in equal strength.
}

$m_{s_{i}}>\mu_{i}^{d}$. Here, $m_{s_{i}}$ are the physical masses of the sterile state $S_{L}$, and $\mu_{i}^{d}$ are the eigenvalues of $\mu$ and $i=1,2$, 3. In the present work, the model parameters are less constrained from the $0 \nu \beta \beta$ decay bound than the unitarity and $m_{s_{i}}>\mu_{i}^{d}$ bounds. The model parameters ranges considered in this work give us $\mathrm{eV}$ to $\mathrm{MeV}$ scale sterile neutrinos as seen by the range of the $m_{s_{1}}$ axis. One interesting thing to note here is that $m_{s_{1}} \geq 10^{-6} \mathrm{GeV}$ is disallowed when we consider both the constraints, unitarity and $0 \nu \beta \beta$. This is mainly due to the unitarity bound since this bound mostly depends on the ratio of $M_{D} / M_{S}$. Therefore, when $\left(M_{D} / M_{S}\right)^{2}<10^{-2}$, those points satisfy the unitarity constraints which are $\pm 10^{-2}$ variations around the unit matrix (see Sec. III B). The disallowed points correspond to a higher ratio, i.e., $\left(M_{D} / M_{S}\right)^{2}>10^{-2}$, and those points represent lower values of the elements of the $\mu$ matrix in order to satisfy the N.O.D which is not covered in Eq. (40). This also implies that the elements of $M_{D}$ and $M_{S}$ are of the same order for the disallowed points and more likely to have higher $M_{S}$ values. Finally, the red points are obtained when we impose the constraint $m_{s_{i}}>\mu_{i}^{d}$. After imposing this constraint, lower values of $m_{s_{1}}$ are getting ruled out which are mostly in the $\mathrm{eV}$ scale. In the right panel of this figure, we have shown the variation of the solar mixing angle $\theta_{12}$ with the lightest sterile neutrino mass. We can see that the whole allowed range of $\theta_{12}$ from oscillation experiments is in agreement with all the constraints.

In the left panel of Fig. 14, we have shown the scatter plot in the $\Delta m_{12}^{2}\left(\mathrm{eV}^{2}\right)-m_{s_{1}}(\mathrm{GeV})$ plane after satisfying the constraints as mentioned in the legend of the figure. Here also, the whole allowed range of $\Delta m_{12}^{2}\left(\mathrm{eV}^{2}\right)$ from oscillation experiments satisfy all constraints. On the other hand, in the right panel, we have shown the scatter plot in the $M_{R 11}-m_{s_{1}}$ plane where $M_{R 11}$ is the eigenvalue of the 

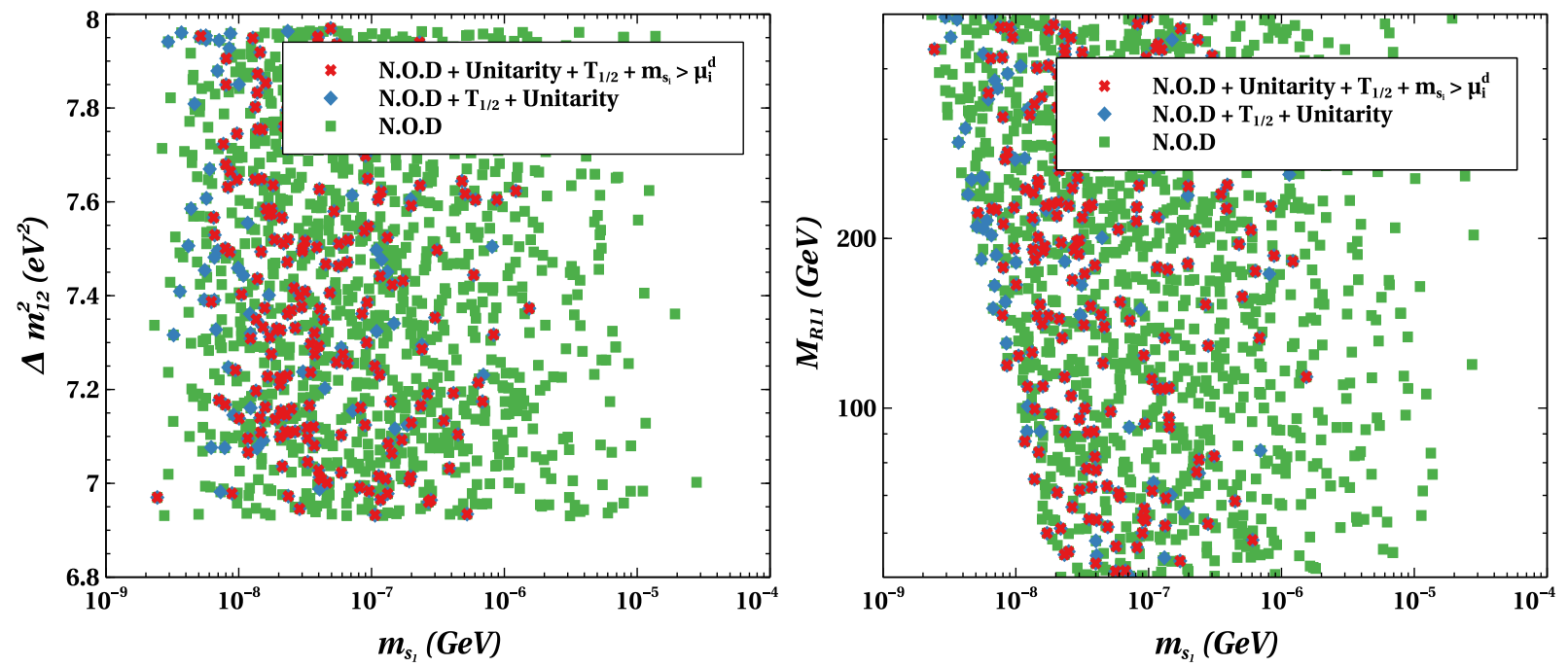

FIG. 14. Scatter plots in $\Delta m_{12}^{2}\left(\mathrm{eV}^{2}\right)-m_{s_{1}}(\mathrm{GeV})$ (left) and $M_{R 11}-m_{s_{1}}$ (both are in $\mathrm{GeV}$ ) (right) planes after satisfying the constraints as mentioned in the text.

$m_{n}$ matrix because we have considered here $M_{R}$ as a diagonal matrix.

The left and right panels of Fig. 15 show the variations in $M_{R 11}$ as a function of $M_{S 11}$ and $M_{S 11}$ as function of $\mu_{11}^{R}$, respectively. Here, the superscript $R$ implies real elements. In both cases, $M_{S 11}>0.02 \mathrm{GeV}$ are not allowed by the unitarity constraint as mentioned earlier. The conditions coming from both unitarity and the seesaw approximation can be respectively manifested as the upper and lower bounds on the light sterile $m_{s}$.

In the left and right panels of Fig. 16, we have shown the scatter plots in plane $M_{D 22}-M_{S 22}$ and $\mu_{23}^{I}(\mathrm{GeV})-\frac{M_{D 11}}{M_{S 11}}$ plane, respectively. Here, the superscript $I$ denotes the imaginary part. In the left panel, we can see that most of the points which satisfy oscillation data are below the yellow line which corresponds to $\frac{M_{D 22}}{M_{S 22}}=10^{-1}$. As we have discussed, the unitarity bound (variation of $\pm 10^{-2}$ around unit matrix) mostly depends on the ratio $\frac{M_{D i i}}{M_{S i i}}(i=1,2,3)$, and we can roughly say if $\frac{M_{D i i}}{M_{S i i}}<10^{-1}$, then it can pass the unitarity bounds. One interesting thing to note here is that there exists a sharp correlation among the $M_{D 22}$ and $M_{S 22}$ parameters (which is valid for other elements of $M_{D}$ and $M_{S}$ matrices also). This is because approximately $\left(M_{D} / M_{S}\right)^{2} \mu \sim 10^{-11} \mathrm{GeV}$, and we have taken $\mu<10^{-8} \mathrm{GeV}$. So $M_{D 22}$ and $M_{S 22}$ cannot take arbitrary values which correspond to significant difference in their magnitudes; otherwise, neutrino mass data will not be
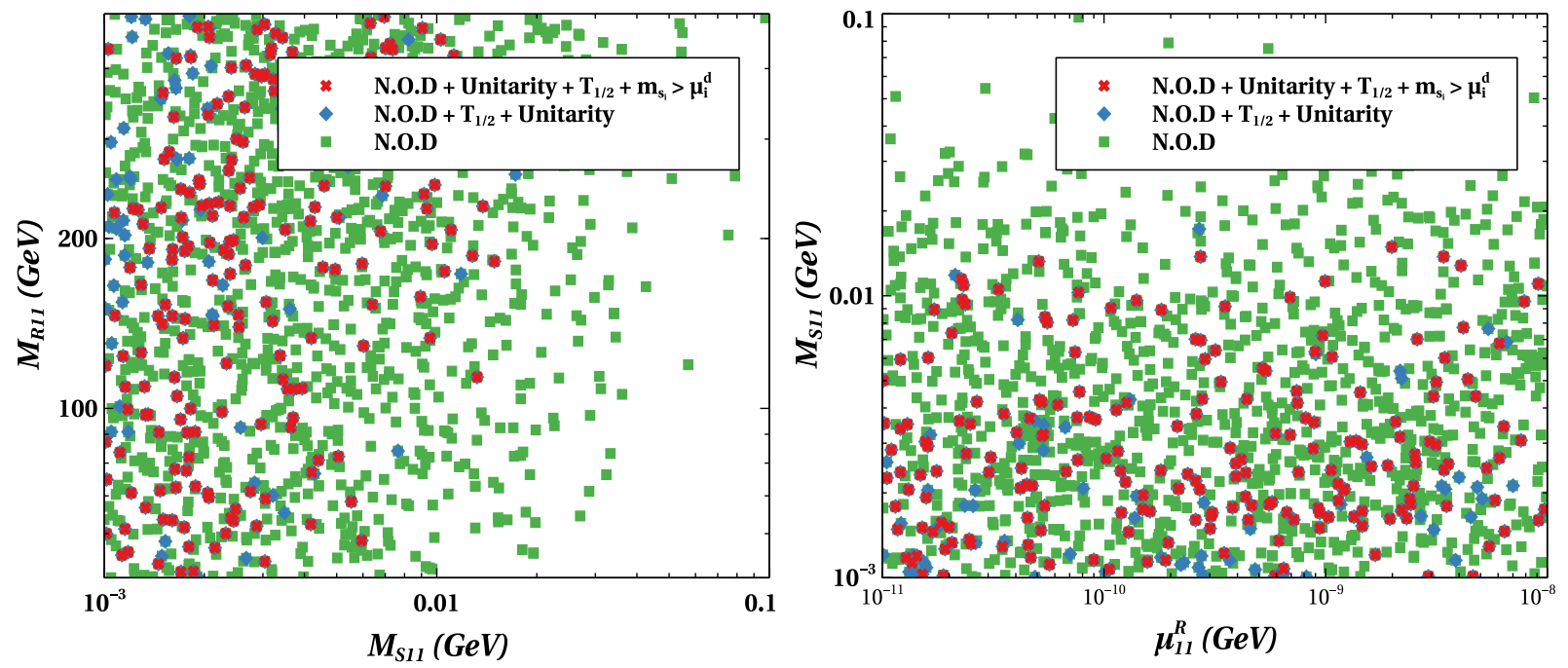

FIG. 15. Scatter plots in $M_{R 11}-M_{S 11}$ (both in $\mathrm{GeV}$ ) (left) and $M_{S 11}-\mu_{11}^{R}$ (both in GeV) (right) planes after satisfying the constraints as mentioned in the text. 

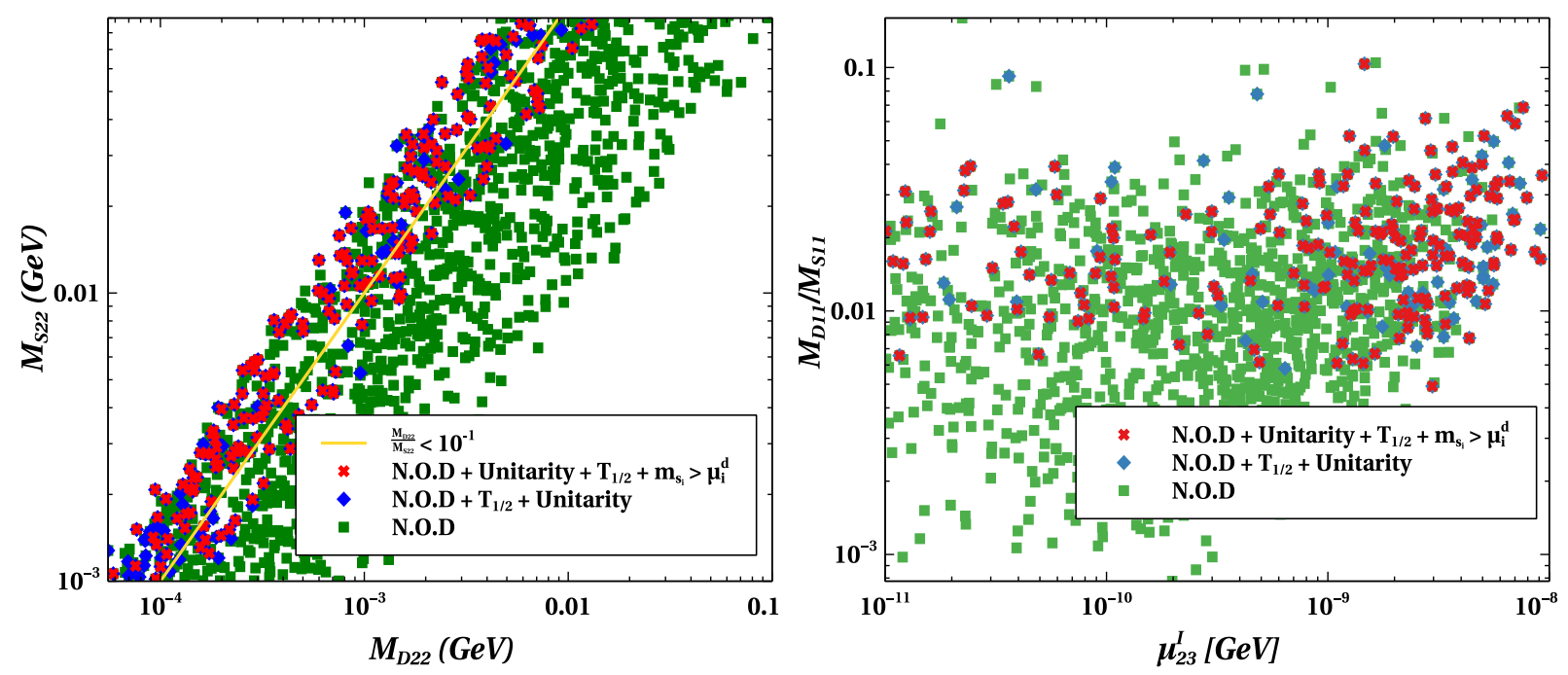

FIG. 16. Scatter plots in $M_{D 22}-M_{S 22}$ (both in $\mathrm{GeV}$ ) (left) and $\mu_{23}^{I}(\mathrm{GeV})-\frac{M_{D 11}}{M_{S 11}}$ (right) planes after satisfying the constraints as mentioned in the text.

satisfied. On the other hand, in the right panel of Fig. 16, we can see that after imposing all the constraints, we get the points which are more prone to having higher values of $\mu_{23}^{I}$. This is because when we impose the unitarity constraint which corresponds to $\frac{M_{D i i}}{M_{S i i}}<10^{-1}(i=1,2,3)$ and from the order of magnitude estimation of neutrino mass, we obtain $\mu \gtrsim 10^{-9}$. This is clearly reflected in the right panel of the figure because the points are more dense in that region where $\mu_{23}^{I} \gtrsim 10^{-9}$ compared to the rest of the region.

\section{CONCLUSION}

In this work, we consider two theory frameworks with sterile neutrinos, (a) left-right symmetric Zee and (b) extended seesaw model, which successfully explain the light neutrino masses and their mixings. Both of the models can accommodate sterile neutrinos with their masses being free parameters varying over a wide range. We particularly focus on a relatively lighter mass range $\sim \mathrm{eV}$ to $\mathrm{MeV}$ and explore the contribution to the $0 \nu \beta \beta$ process. The left-right symmetric Zee model represents a scenario where the masses of the sterile neutrinos are generated at the oneloop level. They are directly dependent on the right-handed Yukawa coupling $\lambda_{R}$ and the masses and mixings of the charged Higgs bosons. For large values of $\lambda_{R}$ (close to the perturbative limit), the sterile neutrino masses always remain well below the $\mathrm{MeV}$ scale. This presents a unique scenario where the three light sterile neutrinos can have significant contributions to the $0 \nu \beta \beta$ process. We find that the half-life of this process crucially depends on three parameters: lightest neutrino mass $m_{\nu_{1}}$, Dirac $C P$ phase $\delta_{C P}$, and $W_{L}-W_{R}$ mixing angle $\theta_{L R}$. In our analysis, we consider the cases with maximal and minimal mixings among the charged Higgs bosons and also consider both the upper and lower values of the NMEs for ${ }^{76} \mathrm{Ge}$ and ${ }^{136} \mathrm{Xe}$ nuclei. The scenario with minimal mixing of the Higgs bosons and maximum values of the NMEs produces the most stringent bound on the model. The calculated half-life for both ${ }^{76} \mathrm{Ge}$ and ${ }^{136} \mathrm{Xe}$ nuclei decreases drastically with an increase in $m_{\nu_{1}}$ or $\theta_{L R}$. This is due to the dominant contributions coming from the $\lambda$ and $\eta$ diagrams as $m_{\nu_{1}}$ and/or $\theta_{L R}$ are increased. This allows us to put quite stringent bounds on both these parameters. For the ${ }^{76} \mathrm{Ge}$ nucleus, the lightest neutrino mass should be less than $10^{-7} \mathrm{eV}$ for $\theta_{L R} \sim 10^{-4}$, while for a lightest neutrino mass of around $10^{-3} \mathrm{eV}$ the value is $\theta_{L R} \lesssim 10^{-8}$, where we consider a normal hierarchy among the active neutrino states. The bounds on the ${ }^{136} \mathrm{Xe}$ nucleus are even more stringent with $m_{\nu_{1}} \lesssim 10^{-8} \mathrm{eV}$ for $\theta_{L R} \sim 10^{-4}$ and $\theta_{L R} \lesssim 10^{-8}$ for $m_{\nu_{1}} \sim 10^{-4} \mathrm{eV}$. Thus, we can significantly constrain the model parameters in this case from the $0 \nu \beta \beta$ studies.

For the extended seesaw, we first consider a onegeneration scenario where in addition to one SM neutrino two sterile neutrinos are also present. Among the two sterile neutrinos, one of them is very heavy with a mass of $10^{5} \mathrm{GeV}$ leading to a negligible contribution in the $0 \nu \beta \beta$ process. The other sterile neutrino has a mass varying between $\mathrm{eV}$ to $\mathrm{MeV}$, and this contributes significantly to the above-mentioned process. We analyze a number of constraints on the model parameters, arising from $0 \nu \beta \beta$, the Daya Bay reactor antineutrino experiment, and nonunitarity constraint on the mixing matrix, as well as theory constraints. We further extend this simplistic one-generation analysis to a higher generation with three active neutrinos and six sterile neutrinos for which we satisfy the neutrino oscillation data. We present a number of correlations between the mass of the lightest sterile neutrino, model parameters, and several neutrino oscillation parameters. In the three-generation case, the masses of the heavy sterile 
states $(N \mathrm{~s})$ have been varied from 50 to $500 \mathrm{GeV}$. These states give negligible contributions to the $0 \nu \beta \beta$ process, while the other three relatively light sterile states $\left(S_{L} \mathrm{~s}\right)$ give substantial contributions. With the considered parameter range, we obtain the upper bound on the mass of the lightest sterile neutrino $S_{1}$ as $10^{-6} \mathrm{GeV}$ after imposing constraints from nonunitarity, $0 \nu \beta \beta$, and others. The nonunitarity of the PMNS matrix has a direct impact on the mixing elements; in extended seesaw, nonunitarity can be governed by the ratio of the bilinear mass term between active neutrino light sterile states $\left(M_{D}\right)$ to the corresponding bilinear mass terms among the sterile states $\left(M_{S}\right)$. The upper bound on the ratio $M_{D} / M_{S}$ is $\sim 10^{-1}$. We choose the effective neutrino mass scale $\left(\mu M_{D}^{2} / M_{S}^{2}\right)$ to be of the order of $10^{-3} \mathrm{eV}$, and we conclude in this scenario the lower bound on the elements of the complex symmetric matrix $\mu$ to be of the order of $10^{-9} \mathrm{GeV}$. Another important constraint in our scenario is $m_{s}>\mu$ below which the seesaw approximation ceases to be valid. It evidently gives a lower bound on $m_{s_{i}}$; i.e., $\mu_{i i}>$ $10^{-9} \mathrm{GeV}$ implies $m_{s_{i}}>1 \mathrm{eV}$. The masses of the other two sterile neutrinos vary as $m_{s_{2,3}} \sim \mathcal{O}(1-10) \mathrm{eV}$.

\section{ACKNOWLEDGMENTS}

The authors acknowledge Ram Lal Awasthi for his contributions at the initial stage of this project. T. J. acknowledges the support from Science and Engineering Research Board (SERB), Government of India, under Grant Reference No. PDF/2020/001053.
[1] I. Esteban, M. C. Gonzalez-Garcia, M. Maltoni, T. Schwetz, and A. Zhou, The fate of hints: Updated global analysis of three-flavor neutrino oscillations, J. High Energy Phys. 09 (2020) 178.

[2] P. Minkowski, $\mu \rightarrow e \gamma$ at a rate of one out of $10^{9}$ muon decays?, Phys. Lett. 67B, 421 (1977).

[3] Proceedings of the Workshop on the Unified Theories and the Baryon Number in the Universe: Tsukuba, Japan, 1979, edited by O. Sawada and A. Sugamoto (National Laboratory for High Energy Physics, Tsukuba, Japan, 1979).

[4] S. Weinberg, Baryon and Lepton Nonconserving Processes, Phys. Rev. Lett. 43, 1566 (1979).

[5] F. Wilczek and A. Zee, Operator Analysis of Nucleon Decay, Phys. Rev. Lett. 43, 1571 (1979).

[6] S. L. Glashow, The future of elementary particle physics, NATO Sci. Ser. B 61, 687 (1980).

[7] M. Gell-Mann, P. Ramond, and R. Slansky, Complex spinors and unified theories, Conf. Proc. C 790927, 315 (1979).

[8] R. N. Mohapatra and G. Senjanovic, Neutrino Mass and Spontaneous Parity Nonconservation, Phys. Rev. Lett. 44, 912 (1980).

[9] A. Zee, Quantum numbers of Majorana neutrino masses, Nucl. Phys. B264, 99 (1986).

[10] K. S. Babu, Model of calculable' Majorana neutrino masses, Phys. Lett. B 203, 132 (1988).

[11] E. Ma, Pathways to Naturally Small Neutrino Masses, Phys. Rev. Lett. 81, 1171 (1998).

[12] F. Bonnet, M. Hirsch, T. Ota, and W. Winter, Systematic study of the $d=5$ Weinberg operator at one-loop order, J. High Energy Phys. 07 (2012) 153.

[13] D. Aristizabal Sierra, A. Degee, L. Dorame, and M. Hirsch, Systematic classification of two-loop realizations of the Weinberg operator, J. High Energy Phys. 03 (2015) 040.

[14] S. K. Kang and C. Kim, Extended double seesaw model for neutrino mass spectrum and low scale leptogenesis, Phys. Lett. B 646, 248 (2007).
[15] M. Mitra, G. Senjanovic, and F. Vissani, Neutrinoless double beta decay and heavy sterile neutrinos, Nucl. Phys. B856, 26 (2012).

[16] R. N. Mohapatra and J. C. Pati, Left-right gauge symmetry and an isoconjugate model of $C P$ violation, Phys. Rev. D 11, 566 (1975).

[17] G. Senjanovic and R. N. Mohapatra, Exact left-right symmetry and spontaneous violation of parity, Phys. Rev. D 12, 1502 (1975).

[18] P. Fileviez Perez and C. Murgui, Lepton flavour violation in left-right theory, Phys. Rev. D 95, 075010 (2017).

[19] S. Khan, M. Mitra, and A. Patra, Neutrino and collider implications of a left-right extended Zee model, Phys. Rev. D 98, 115038 (2018).

[20] A. Zee, A theory of lepton number violation, neutrino Majorana mass, and oscillation, Phys. Lett. 93B, 389 (1980); Erratum, Phys. Lett. 95B, 461 (1980).

[21] P. H. Frampton, M. C. Oh, and T. Yoshikawa, Zee model confronts SNO data, Phys. Rev. D 65, 073014 (2002).

[22] Y. Koide, Prospect of the Zee model, Nucl. Phys. B Proc. Suppl. 111, 294 (2002).

[23] X.-G. He, Is the Zee model neutrino mass matrix ruled out?, Eur. Phys. J. C 34, 371 (2004).

[24] P. Fileviez Perez, C. Murgui, and S. Ohmer, Simple leftright theory: Lepton number violation at the LHC, Phys. Rev. D 94, 051701 (2016).

[25] I. Girardi, A. Meroni, and S. T. Petcov, Neutrinoless double beta decay in the presence of light sterile neutrinos, J. High Energy Phys. 11 (2013) 146.

[26] G. Karagiorgi, Toward solution of the MiniBooNE-LSND anomalies, Nucl. Phys. B Proc. Suppl. 229-232, 50 (2012).

[27] M. Antonello et al., Experimental search for the "LSND anomaly" with the ICARUS detector in the CNGS neutrino beam, Eur. Phys. J. C 73, 2345 (2013).

[28] A. Aguilar-Arevalo et al. (LSND Collaboration), Evidence for neutrino oscillations from the observation of $\bar{\nu}_{e}$ appearance in a $\bar{\nu}_{\mu}$ beam, Phys. Rev. D 64, 112007 (2001). 
[29] A. Aguilar-Arevalo et al. (MiniBooNE Collaboration), Unexplained Excess of Electron-Like Events From a 1GeV Neutrino Beam, Phys. Rev. Lett. 102, 101802 (2009).

[30] B. Armbruster et al. (KARMEN Collaboration), Upper limits for neutrino oscillations muon-anti-neutrino $\longrightarrow$ electron-anti-neutrino from muon decay at rest, Phys. Rev. D 65, 112001 (2002).

[31] P. Adamson et al. (MINOS Collaboration), Search for Sterile Neutrinos Mixing with Muon Neutrinos in MINOS, Phys. Rev. Lett. 117, 151803 (2016).

[32] B. Abi et al. (DUNE Collaboration), The DUNE far detector interim design report volume 1: Physics, technology and strategies, arXiv:1807.10334.

[33] B. Abi et al. (DUNE Collaboration), Deep Underground Neutrino Experiment (DUNE), far detector technical design report, volume II: DUNE physics, arXiv:2002 .03005 .

[34] G. Mention, M. Fechner, T. Lasserre, T. Mueller, D. Lhuillier, M. Cribier, and A. Letourneau, The reactor antineutrino anomaly, Phys. Rev. D 83, 073006 (2011).

[35] K. N. Abazajian et al., Light sterile neutrinos: A white paper, arXiv:1204.5379.

[36] F. Kaether, W. Hampel, G. Heusser, J. Kiko, and T. Kirsten, Reanalysis of the GALLEX solar neutrino flux and source experiments, Phys. Lett. B 685, 47 (2010).

[37] J. Abdurashitov et al. (SAGE Collaboration), Measurement of the solar neutrino capture rate with gallium metal. III: Results for the 2002-2007 data-taking period, Phys. Rev. C 80, 015807 (2009).

[38] M. Drewes et al., A white paper on $\mathrm{keV}$ sterile neutrino dark matter, J. Cosmol. Astropart. Phys. 01 (2017) 025.

[39] N. Sabti, J. Alvey, M. Escudero, M. Fairbairn, and D. Blas, Refined bounds on $\mathrm{MeV}$-scale thermal dark sectors from BBN and the CMB, J. Cosmol. Astropart. Phys. 01 (2020) 004.

[40] E. Majorana, Teoria simmetrica dell'elettrone e del positrone, Nuovo Cimento 14, 171 (1937).

[41] G. Racah, On the symmetry of particle and antiparticle, Nuovo Cimento 14, 322 (1937).

[42] W. H. Furry, On transition probabilities in double betadisintegration, Phys. Rev. 56, 1184 (1939).

[43] J. D. Vergados, H. Ejiri, and F. Šimkovic, Neutrinoless double beta decay and neutrino mass, Int. J. Mod. Phys. E 25, 1630007 (2016).

[44] S. Dell'Oro, S. Marcocci, M. Viel, and F. Vissani, Neutrinoless double beta decay: 2015 review, Adv. High Energy Phys. 2016, 2162659 (2016).

[45] M. Agostini et al. (GERDA Collaboration), Improved Limit on Neutrinoless Double- $\beta$ Decay of ${ }^{76} \mathrm{Ge}$ from GERDA Phase II, Phys. Rev. Lett. 120, 132503 (2018).

[46] A. Gando et al. (KamLAND-Zen Collaboration), Search for Majorana Neutrinos near the Inverted Mass Hierarchy Region with KamLAND-Zen, Phys. Rev. Lett. 117, 082503 (2016); 117, 109903(A) (2016).

[47] C. Arnaboldi et al. (CUORE Collaboration), CUORE: A Cryogenic underground observatory for rare events, Nucl. Instrum. Methods Phys. Res., Sect. A 518, 775 (2004).

[48] R. Arnold et al. (SuperNEMO Collaboration), Probing new physics models of neutrinoless double beta decay with SuperNEMO, Eur. Phys. J. C 70, 927 (2010).
[49] P. A. R. Ade et al. (Planck Collaboration), Planck 2015 results. XIII. Cosmological parameters, Astron. Astrophys. 594, A13 (2016).

[50] M. Blennow, P. Coloma, E. Fernandez-Martinez, J. Hernandez-Garcia, and J. Lopez-Pavon, Non-unitarity, sterile neutrinos, and non-standard neutrino interactions, J. High Energy Phys. 04 (2017) 153.

[51] T. Asaka, S. Eijima, and H. Ishida, Mixing of active and sterile neutrinos, J. High Energy Phys. 04 (2011) 011.

[52] J. T. Penedo and S. T. Petcov, The $10^{-3} \mathrm{eV}$ frontier in neutrinoless double beta decay, Phys. Lett. B 786, 410 (2018).

[53] J. Herrero-García, T. Ohlsson, S. Riad, and J. Wirén, Full parameter scan of the Zee model: Exploring Higgs lepton flavor violation, J. High Energy Phys. 04 (2017) 130.

[54] G. Ecker, W. Grimus, and H. Neufeld, Higgs induced flavor changing neutral interactions in SU(2)-1 X SU(2)-r X U(1), Phys. Lett. 127B, 365 (1983); Erratum, Phys. Lett. 132B, 467 (1983).

[55] R. N. Mohapatra, G. Senjanovic, and M. D. Tran, Strangeness changing processes and the limit on the right-handed gauge boson mass, Phys. Rev. D 28, 546 (1983).

[56] M. E. Pospelov, FCNC in left-right symmetric theories and constraints on the right-handed scale, Phys. Rev. D 56, 259 (1997).

[57] Y. Zhang, H. An, X. Ji, and R. N. Mohapatra, General CP violation in minimal left-right symmetric model and constraints on the right-handed scale, Nucl. Phys. B802, 247 (2008).

[58] A. Maiezza, M. Nemevsek, F. Nesti, and G. Senjanovic, Left-right symmetry at LHC, Phys. Rev. D 82, 055022 (2010).

[59] D. Guadagnoli and R. N. Mohapatra, TeV scale left right symmetry and flavor changing neutral Higgs effects, Phys. Lett. B 694, 386 (2011).

[60] J. Barry and W. Rodejohann, Lepton number and flavour violation in $\mathrm{TeV}$-scale left-right symmetric theories with large left-right mixing, J. High Energy Phys. 09 (2013) 153.

[61] R. L. Awasthi, M. K. Parida, and S. Patra, Neutrino masses, dominant neutrinoless double beta decay, and observable lepton flavor violation in left-right models and $\mathrm{SO}(10)$ grand unification with low mass $W_{R}, Z_{R}$ bosons, J. High Energy Phys. 08 (2013) 122.

[62] J. Chakrabortty, H. Z. Devi, S. Goswami, and S. Patra, Neutrinoless double- $\beta$ decay in TeV scale Left-Right symmetric models, J. High Energy Phys. 08 (2012) 008.

[63] P. S. Bhupal Dev, S. Goswami, and M. Mitra, TeV scale left-right symmetry and large mixing effects in neutrinoless double beta decay, Phys. Rev. D 91, 113004 (2015).

[64] J. Chakrabortty, J. Gluza, R. Sevillano, and R. Szafron, Left-right symmetry at LHC and precise 1-loop low energy data, J. High Energy Phys. 07 (2012) 038.

[65] G. Aad et al. (ATLAS Collaboration), Search for heavy Majorana neutrinos with the ATLAS detector in pp collisions at $\sqrt{s}=8 \mathrm{TeV}$, J. High Energy Phys. 07 (2015) 162.

[66] U. Kaya, M. Sahin, and S. Sultansoy, Majorana neutrino and $W_{R}$ at TeV scale $e p$ colliders, arXiv:1502.04115. 
[67] N. Arkani-Hamed, T. Han, M. Mangano, and L.-T. Wang, Physics opportunities of a $100 \mathrm{TeV}$ proton-proton collider, Phys. Rep. 652, 1 (2016).

[68] T. Golling et al., Physics at a $100 \mathrm{TeV}$ pp collider: Beyond the Standard Model phenomena, CERN Yellow Rep. 3, 441 (2017).

[69] M. Mitra, R. Ruiz, D. J. Scott, and M. Spannowsky, Neutrino jets from high-mass $W_{R}$ gauge bosons in TeVscale left-right symmetric models, Phys. Rev. D 94, 095016 (2016).

[70] D. Borah and A. Dasgupta, Naturally light dirac neutrino in left-right symmetric model, J. Cosmol. Astropart. Phys. 06 (2017) 003.

[71] Y. Cai, T. Han, T. Li, and R. Ruiz, Lepton number violation: Seesaw models and their collider tests, Front. Phys. 6, 40 (2018).

[72] J. Beringer et al. (Particle Data Group Collaboration), Review of Particle Physics (RPP), Phys. Rev. D 86, 010001 (2012).

[73] J.A. Casas and A. Ibarra, Oscillating neutrinos and $\mu \rightarrow e, \gamma$, Nucl. Phys. B618, 171 (2001).

[74] G. Pantis, F. Simkovic, J. D. Vergados, and A. Faessler, Neutrinoless double beta decay within QRPA with proton-neutron pairing, Phys. Rev. C 53, 695 (1996).

[75] J. Suhonen and O. Civitarese, Weak-interaction and nuclear-structure aspects of nuclear double beta decay, Phys. Rep. 300, 123 (1998).

[76] V. A. Rodin, A. Faessler, F. Simkovic, and P. Vogel, On the uncertainty in the $0 \mathrm{nu}$ beta beta decay nuclear matrix elements, Phys. Rev. C 68, 044302 (2003).

[77] V. A. Rodin, A. Faessler, F. Simkovic, and P. Vogel, Assessment of uncertainties in QRPA $0 \nu \beta \beta$-decay nuclear matrix elements, Nucl. Phys. A766, 107 (2006); A793, 213(E) (2007).

[78] F. Simkovic, A. Faessler, V. Rodin, P. Vogel, and J. Engel, Anatomy of nuclear matrix elements for neutrinoless double-beta decay, Phys. Rev. C 77 (2008) 045503.

[79] F. Simkovic, A. Faessler, H. Muther, V. Rodin, and M. Stauf, The $0 \mathrm{nu}$ bb-decay nuclear matrix elements with self-consistent short-range correlations, Phys. Rev. C 79, 055501 (2009).

[80] J. Kotila and F. Iachello, Phase space factors for double- $\beta$ decay, Phys. Rev. C 85, 034316 (2012).

[81] A. Dueck, W. Rodejohann, and K. Zuber, Neutrinoless double beta decay, the inverted hierarchy and precision determination of theta(12), Phys. Rev. D 83, 113010 (2011).

[82] A. Faessler, M. González, S. Kovalenko, and F. Šimkovic, Arbitrary mass Majorana neutrinos in neutrinoless double beta decay, Phys. Rev. D 90, 096010 (2014).

[83] W. Dekens, J. de Vries, K. Fuyuto, E. Mereghetti, and G. Zhou, Sterile neutrinos and neutrinoless double beta decay in effective field theory, J. High Energy Phys. 06 (2020) 097.

[84] J. Alcaide, D. Das, and A. Santamaria, A model of neutrino mass and dark matter with large neutrinoless double beta decay, J. High Energy Phys. 04 (2017) 049.

[85] O. Cremonesi, Neutrinoless double beta decay, J. Phys. Conf. Ser. 718, 022006 (2016).
[86] T. Peng, M. J. Ramsey-Musolf, and P. Winslow, TeV lepton number violation: From neutrinoless double- $\beta$ decay to the LHC, Phys. Rev. D 93, 093002 (2016).

[87] E. Lisi, A. Rotunno, and F. Simkovic, Degeneracies of particle and nuclear physics uncertainties in neutrinoless $\beta \beta$ decay, Phys. Rev. D 92, 093004 (2015).

[88] P. S. Bhupal Dev, C.-H. Lee, and R. N. Mohapatra, TeV scale lepton number violation and baryogenesis, J. Phys. Conf. Ser. 631, 012007 (2015).

[89] S. Pascoli, M. Mitra, and S. Wong, Effect of cancellation in neutrinoless double beta decay, Phys. Rev. D 90, 093005 (2014).

[90] W.-C. Huang and J. Lopez-Pavon, On neutrinoless double beta decay in the minimal left-right symmetric model, Eur. Phys. J. C 74, 2853 (2014).

[91] P. S. Bhupal Dev, S. Goswami, M. Mitra, and W. Rodejohann, Constraining neutrino mass from neutrinoless double beta decay, Phys. Rev. D 88, 091301 (2013).

[92] S. Khan, S. Goswami, and S. Roy, Vacuum stability constraints on the minimal singlet $\mathrm{TeV}$ seesaw model, Phys. Rev. D 89, 073021 (2014).

[93] C. F. Wong, Phenomenology of sterile nutrinos at different mass scales: Neutrinoless double beta decay and neutrino oscillations, Ph.D. thesis, Durham University, 2012.

[94] J. Chakrabortty, M. Das, and S. Mohanty, Constraints on $\mathrm{TeV}$ scale Majorana neutrino phenomenology from the Vacuum Stability of the Higgs, Mod. Phys. Lett. A 28, 1350032 (2013).

[95] M. Mitra, G. Senjanovic, and F. Vissani, Heavy sterile neutrinos and neutrinoless double beta decay, in Proceedings of the 47th Rencontres de Moriond on Electroweak Interactions and Unified Theories (2012), https:// inspirehep.net/literature/1268713.

[96] J. D. Vergados, H. Ejiri, and F. Simkovic, Theory of neutrinoless double beta decay, Rep. Prog. Phys. 75 (2012) 106301.

[97] R. N. Mohapatra, Mechanism for Understanding Small Neutrino Mass in Superstring Theories, Phys. Rev. Lett. 56, 561 (1986).

[98] R. N. Mohapatra and J. W. F. Valle, Neutrino mass and baryon number nonconservation in superstring models, Phys. Rev. D 34, 1642 (1986).

[99] D. Wyler and L. Wolfenstein, Massless neutrinos in leftright symmetric models, Nucl. Phys. B218, 205 (1983).

[100] E. Witten, New issues in manifolds of SU(3) holonomy, Nucl. Phys. B268, 79 (1986).

[101] J. L. Hewett and T. G. Rizzo, Low-energy phenomenology of superstring inspired E(6) models, Phys. Rep. 183, 193 (1989).

[102] P. S. B. Dev and R. N. Mohapatra, TeV scale inverse seesaw in $\mathrm{SO}(10)$ and leptonic non-unitarity effects, Phys. Rev. D 81, 013001 (2010).

[103] S. Blanchet, P. S. B. Dev, and R. N. Mohapatra, Leptogenesis with $\mathrm{TeV}$ scale inverse seesaw in SO(10), Phys. Rev. D 82, 115025 (2010).

[104] A. Ilakovac and A. Pilaftsis, Flavor violating charged lepton decays in seesaw-type models, Nucl. Phys. B437, 491 (1995).

[105] F. Deppisch and J. W. F. Valle, Enhanced lepton flavor violation in the supersymmetric inverse seesaw model, Phys. Rev. D 72, 036001 (2005). 
[106] C. Arina, F. Bazzocchi, N. Fornengo, J. C. Romao, and J. W. F. Valle, Minimal Supergravity Sneutrino Dark Matter and Inverse Seesaw Neutrino Masses, Phys. Rev. Lett. 101, 161802 (2008).

[107] M. Malinsky, T. Ohlsson, Z.-z. Xing, and H. Zhang, Nonunitary neutrino mixing and $C P$ violation in the minimal inverse seesaw model, Phys. Lett. B 679, 242 (2009).

[108] M. Hirsch, T. Kernreiter, J. C. Romao, and A. Villanova del Moral, Minimal supersymmetric inverse seesaw: Neutrino masses, lepton flavour violation and LHC phenomenology, J. High Energy Phys. 01 (2010) 103.

[109] P. F. de Salas, D. V. Forero, S. Gariazzo, P. MartínezMiravé, O. Mena, C. A. Ternes, M. Tórtola, and J. W. F. Valle, 2020 global reassessment of the neutrino oscillation picture, J. High Energy Phys. 02 (2021) 071.

[110] F. P. An et al. (Daya Bay Collaboration), Improved Search for a Light Sterile Neutrino with the Full Configuration of the Daya Bay Experiment, Phys. Rev. Lett. 117, 151802 (2016).

[111] A. Abada, V. De Romeri, and A. M. Teixeira, Effect of steriles states on lepton magnetic moments and neutrinoless double beta decay, J. High Energy Phys. 09 (2014) 074.

[112] J. C. Helo, S. Kovalenko, and I. Schmidt, Sterile neutrinos in lepton number and lepton flavor violating decays, Nucl. Phys. B853, 80 (2011).

[113] S. Kovalenko, Z. Lu, and I. Schmidt, Lepton number violating processes mediated by Majorana neutrinos at hadron colliders, Phys. Rev. D 80, 073014 (2009).

[114] A. Meroni, S. T. Petcov, and F. Simkovic, Multiple $C P$ non-conserving mechanisms of $(\beta \beta)_{0 \nu}$-decay and nuclei with largely different nuclear matrix elements, J. High Energy Phys. 02 (2013) 025. 